\title{
Fusing passive RFID and BIM for increased accuracy in indoor localization
}

\author{
Aaron M. Costin ${ }^{1}$ and Jochen Teizer ${ }^{2^{*}}$
}

\begin{abstract}
Background: Finding the current location of a specific utility or oneself in an unfamiliar facility can be difficult and time consuming. The hypothesis tested in this paper is that using the information contained within Building Information Models (BIM) can increase the accuracy of indoor positioning algorithms using context-aware sensing technology. The presented work demonstrates how the integration of passive Radio Frequency Identification (RFID) tracking technology and Building Information Modeling (BIM) can assist indoor localization for potential applications in facilities management for proactive preventative maintenance.

Methods: This paper includes (1) developing a framework that utilizes the integration of commercially-available RFID and a building information model; (2) evaluating the framework for real-time resource location tracking within an indoor environment; and (3) developing an algorithm for real-time localization and visualization in a BIM. A prototype application has been developed that simultaneously connects the RFID readers on a maintenance cart, an asset maintenance database and a BIM model. Three multilateralization approaches were compared in the system to use in the algorithm. Testing was conducted in a facility with a corridor that loops around in a rectangle.

Results: The goal is to have a system accuracy within $3 \mathrm{~m}$. Results show that fusing BIM with multilateralization techniques for RFID technology can decrease the number of false reads by $64 \%$ versus standalone multilateralization equations. The greatest system accuracy achieved was $1.66 \mathrm{~m}$.

Conclusions: Significantly, the results validate the hypothesis that BIM can increase indoor localization accuracy, and show the usefulness of using BIM for indoor localization in addition to real-time visualization.

Keywords: Building information modeling (BIM), Context aware information, Facility management, Indoor localization, Radio frequency identification (RFID)
\end{abstract}

\section{Introduction}

The operation and maintenance $(\mathrm{O} \& M)$ phase of a building deals with management and maintenance of assets in the building for smooth functioning. Technology and innovation has been shown to improve the functionality, sustainability and flexibility of facility components (Olatunji and Sher 2009). Additionally, building systems are becoming increasingly complex, causing challenges for the management and operation of the facility (Kean 2011). Maintenance is an on-going process that runs throughout the project life cycle and requires continuous navigation throughout the facility. Facility management (FM) relies heavily on the constant monitoring of

\footnotetext{
* Correspondence: jochen@teizer.de

${ }^{2}$ RAPIDS Construction Safety and Technology Laboratory, Ettlingen, Germany Full list of author information is available at the end of the article
}

numerous items located throughout the building to be proactive when it comes to preventative maintenance.

The process of manual inspection proves to be time consuming as it relies distinctly on a worker searching throughout a facility for problems often without precise information relating to location. Manually inspecting or locating various building components causes workers to spend excessive amounts of time searching for the desired equipment or materials, rather than working efficiently on the tasks required for proper maintenance. It has been shown that locating equipment in facilities is the core maintenance activity that causes significant delay in maintenance (Lee and Akin 2009). Moreover, locating building components is critical for timely repair of the component and mitigation of the damage (Taneja et al. 2012).

\section{实 Springer}

(c) 2015 Costin and Teizer. Open Access This article is distributed under the terms of the Creative Commons Attribution 4.0 International License (http://creativecommons.org/licenses/by/4.0/), which permits unrestricted use, distribution, and reproduction in any medium, provided you give appropriate credit to the original author(s) and the source, provide a link to the Creative Commons license, and indicate if changes were made. 
Through the use of context-aware, e.g., location, time and three-dimensional (3D) space based, automated systems, this process can be greatly improved to increase the performance of facilities management. The need for such technologies in an indoor setting is crucial since research has shown that $85 \%$ of the total project cost is spent in operation and maintenance from the owner's perspective (Teicholz 2004).

Real-time access to the locations of workers, materials and equipment has become a significant advancement to the management of construction processes. Location-aware computing offers significant potential of improving such manual processes and supporting important decision making tasks in the field (Teizer et al. 2007). There have been a variety of technologies (e.g., Ultra Wideband, Global Positioning System, laser scanner) utilized to produce visualizations of the locations of resources on a construction site (Khoury and Kamat 2009, Pradhananga and Teizer 2013, Vasenev et al. 2014, Pradhananga and Teizer 2015, Cheng et al. 2013c, Cheng et al. 2013d). However, there is a lack of real-time data visualization of such technologies within an indoor environment to determine a person's location inside the facility, as well as an aid for navigation (Teizer et al. 2013, Cheng and Teizer 2013, b).

One solution is the integration of emerging wireless remote sensing data with Building Information Models (BIM) which allows for the real-time visualization of the locations of workers, materials and equipment. Integrated building technologies allow a convergence and integration of systems to play a greater role in overall building performance (Kean 2011). Unfortunately, little research has been conducted regarding the reliability and practical benefits of the integration. The development of a building model holds large potentials in saving time, reducing miscommunications and enabling internet-based building/FM integration and interoperability, but it has yet to evolve to a point where it incorporates the needs of FM (Teicholz 2004).

A building information model can contain an abundance of information about that facility. The purpose is to utilize that information stored within the BIM model to increase the accuracy of localization algorithms. This paper describes what information can be utilized, such as the spatial information (corridors, size of components, coordinates, etc.), building objects contained in the model (walls, doors, HVAC, pipes, etc.) and attributes associated with each object (object type, object properties, linked documentation, etc.). Additionally, that 3D model can be used for real-time visualization, which has a variety of benefits.

\section{Background}

In an industry where time is crucial for remaining on schedule and lowering FM costs, manual inspection remains especially inefficient and costly (Razavi et al. 2012). Current methods of planning and executing FM are based on personal knowledge and experience (Akcamete et al. 2010). Additionally, paper-based quality inspections involve labor-intensive methods and are shown to be unreliable and time consuming (Wang 2008). Fig. 1 shows a FM employee using paper-based methods to locate a desired utility, which can be time consuming and add a delay in the maintenance task.

As a study has shown, more than half of all maintenance resources and activities of a facility are reactive

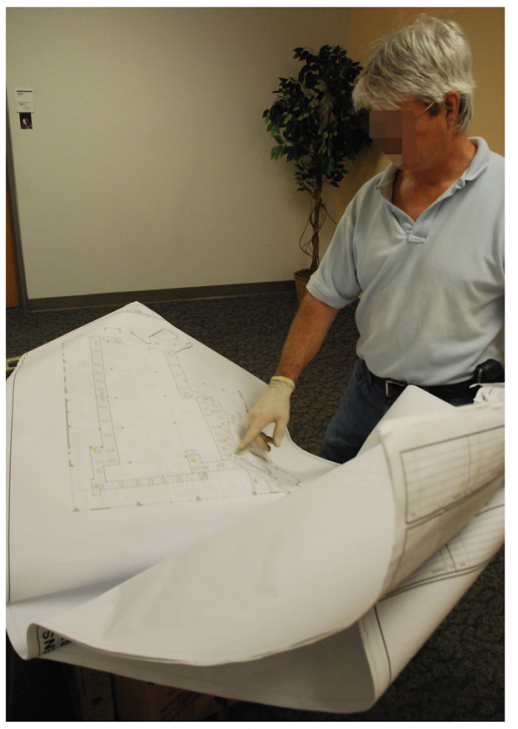

(a)

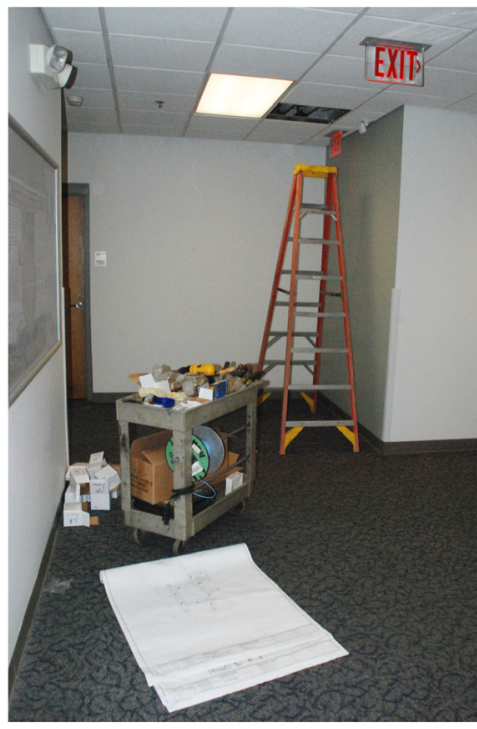

(b)

Fig. 1 Facilities management: (a) facilities manager locating utility on a paper map and (b) facilities manager's maintenance cart and equipment 
maintenance, which is to wait until a utility breaks before it is serviced (Sullivan et al. 2010). The most cost effective maintenance is preventative, in which actions are performed on a time-based schedule with the aim of sustaining or extending a resource's useful life through controlling degradation to an acceptable level (Sullivan et al. 2010).

Automated systems have the potential to eliminate manual tasks (e.g., safety and inspection documents, warranties) and provide the ability for more cost-effective preventative maintenance (e.g. real-time scheduling, maintenance histories) (Thomas et al. 2012). For instance, they can alert the facility manager when a building component is due for an inspection and provide the digital details of that component, such as the maintenance history including the location within the facility, which are commonly marked up on paper documents. Moreover, integrating such systems with context-aware information provides potential for improvement in working practices, particularly with respect to productivity and safety (Anumba and Aziz 2006).

\section{Context-aware information}

A context-aware system is a system that automatically recognizes its location and surroundings or provides context-aware information that locates other objects. Context-aware information has promising benefits including, through sensing technology and BIM, near real-time access to location and spatial (3D) relationships to utilities for FM, respectively. However, currently available FM systems are not fully benefiting from the 3D visualization capabilities of BIMs and the information of topological relationships between components in order to support spatial analyses of maintenance information (Akcamete et al. 2010).

Circumstances may arise where poor visibility makes detection of utilities difficult for a facility manager, causing problems to remain unnoticed and resources to remain inoperative.] Moreover, workers intending to correct an issue but who are unfamiliar with a facility may have difficultly locating themselves, as well as locating a specific room within a facility. It is also of utmost importance that the facility can be navigated quickly in the event of an emergency, because a search and rescue crew has no time to waste in getting lost when human lives are at stake. A context-aware system can provide occupants, personnel or emergency crews with location information to navigate around and find their destinations ( $\mathrm{Li}$ et al. 2011). Therefore, the hypothesis is that integrating real-time automated location sensing technology and building information modeling would provide real time visualization that can assist in localization and navigation of a facility. Additionally, the system would provide context-aware information to help increase preventative maintenance and minimize manual tasks.

\section{Radio frequency identification technology}

Radio Frequency Identification (RFID) is the wireless communication via radio waves that uses three main components in this system: a computer, an interrogator and a tag (Fig. 2). The interrogator (also known as a reader) continuously broadcasts a RF (radio frequency) signal through the antenna. Any tag (also known as a responder) within the RF field is an activated tag that rebroadcasts a signal along with the unique data stored on the tag. The reader then captures the signal from the tag and transfers the data to the computer where it is processed through middleware and stored in a database. Figure 2 also displays a wireless RFID system, in which the interrogator has WiFi capabilities to communicate to a wireless router connected to the computer. Significantly, the tag data that the reader transfers to the computer can be utilized in many ways by querying the database for the tag's unique ID number. Essentially, when a tag is read all the information about that tag object can be accessed, i.e. unit name, installation date, manufacturer and safety instructions (Thomas et al. 2012).

RFID systems are classified as either active (powered by battery). passive (powered by RF wave), or semiactive/passive (both). RFID is similar to barcoding, but removes the human component of physically scanning each barcode. While bar code systems are limited due to
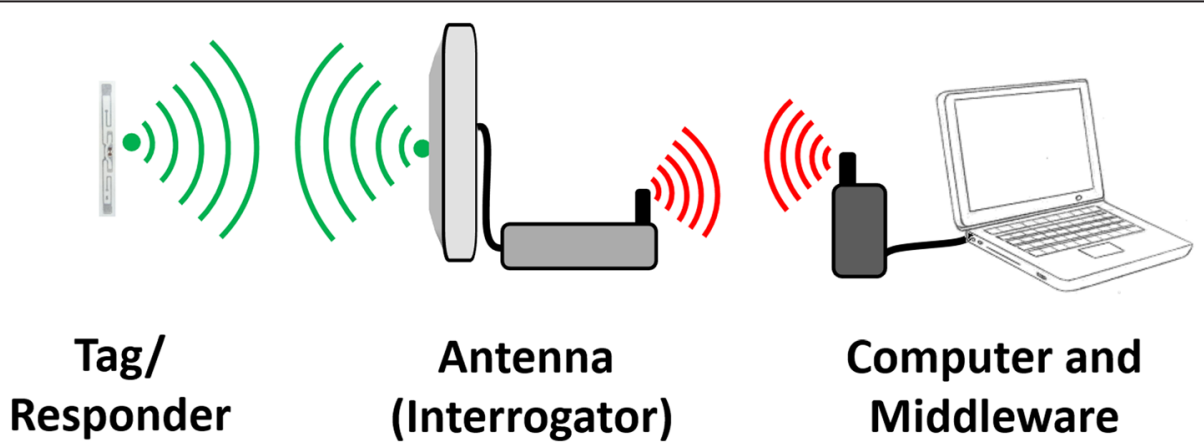

Computer and Middleware

Fig. 2 Components of a wireless RFID system 
line-of-sight, durability and read-range constraints, passive ultrahigh RFID provides somewhat greater readranges and works under rugged outdoor and indoor conditions, including in temperatures from $-40{ }^{\circ} \mathrm{C}$ to $200{ }^{\circ} \mathrm{C}$, extreme moisture, $\mathrm{pH}$ and pressure (Vogt and Teizer 2007, Ross et al. 2009).

Passive RFID tags do not transmit power, but rather reflect the power back to the antenna, called incident power. In order for a passive tag to operate, the reader must supply the operating energy to the tag through the transmission of an RF wave. The output power is the energy the reader sends out. The tag then reflects the signal back (consuming some in the process). The Received Signal Strength Indicator (RSSI) is the measurement of how much power is received by the reader from the tag, relative to the original signal strength from the reader. The signal strength propagates and reduces when the distance between the antenna and tag is increased. The further away a tag is, the lower the RSSI. The maximum RSS is the power output from the reader, and the minimum is the signal strength needed to operate the tag. Therefore, knowing the output power from the antenna and the performance characteristics of the antennas and tags, an approximation of the tag location can be made.

The Friis transmission equation (Ahson and Ilyas 2008, Wang et al. 2009) is used to calculate the power received by an antenna, when transmitted from another antenna. Free-space path loss is proportional to the square of the distance between the transmitter and receiver. Additionally, path loss is also proportional to the square of the frequency of the radio signal. Tracing the power to and from the reader can provide insights into both tag detection and the RSSI. The key elements involving physical aspects of antennas are antenna gains, reflection coefficients and polarization. However, in real world applications, other factors include the effects of impedance mismatch, misalignment of the antenna pointing, atmospheric conditions, absorption, shadowing, diffraction and material properties.

\section{Building information modeling}

Building Information Modeling (BIM) has become a paradigm shift from the traditional design approach in the Architecture, Engineering and Construction (AEC) industry. BIM is a process that encompasses the lifecycle of a building and allows for the physical and functional characteristics of a building to be designed, modeled and managed. Through BIM, an accurate virtual digital model of a project is constructed, which has been found to be one of the most promising advancement in the AEC industry (Eastman et al. 2011). Paired with sensing technologies, the model can provide the spatial relationships needed for context-aware information (Costin et al. 2012a). In addition, each component of the model, their relationship with other objects in the model and logical classification of objects in the model, are stored directly within the model. Integration of all the aspects on the projects like structural, architectural, mechanical and electrical plumbing (MEP), energy and more into the same platform has enabled a new era of collaboration for better design and optimized performance. These benefits have led to a dramatic increase in the use of BIM over the years, in which $71 \%$ of construction companies, $70 \%$ of architects, and $74 \%$ of contractors are using BIM, and the use is expected to keep increasing (McGraw-Hill 2012).

The universal circulation network (UCN) presented in Lee et al. (2010)) provides a method for representing circulation paths from a BIM model. Utilizing the spatial topology and geometry of the model, the UCN can determine walking distances, including the shortest and most efficient. The ability to calculate circulation paths holds enormous potential for indoor localization and visualization, especially if linked to remote sensing technologies. Making uses of the industry foundation classes (IFCs) (buildingSMART 2013) the model provides two key pieces of spatial information: (1) the coordinates of objects, i.e., IfcCartesianPoint, needed for localization and (2) the topology and geometry, i.e. IfcSpace, needed to navigate the building (Costin and Teizer 2014).

There have been studies conducted involving the integration of RFID and BIM. A study by Xie and Shi (Xie et al. 2010) focused on using GPS and RFID for tagging steel components and using the same unique tag for each component throughout the BIM model in the project. Updating the status in the BIM model would automatically keep track of the components from delivery to installation. RFID technology has also been utilized by successfully pairing with Building Information Modeling (BIM) for supply chain management (Sawyer 2014). An integration of RFID and BIM has been proposed for life cycle information management of open-buildings (Cheng and Chang 2011), where the integration in planning and design, manufacturing, construction and maintenance, as well as recycle and reuse is discussed. Li et al. (2014) integrated a BIM centric algorithm for fire emergency response. A pilot study has been performed using RFID for FM based on a BIM database (Meadati et al. 2010) to determine the feasibility of enabling an automate information exchange environment. The pilot study does conclude that the RFID-BIM integration provides seamless information exchange between the real world components and respective BIM components, but lacks the real-time indoor localization and visualization components. Therefore, research focusing on using an intelligent model for FM with real-time indoor localization and visualization has been lacking. 


\section{Facility management}

FM requires a robust database linked to the visualization environment for identifying the items within. Currently, BIM has been used as a digital copy of the facility which is independent of the actual facility. Becerik-Gerber et al. (2012) researched the integration of BIM with facilities management. The findings conclude that BIM holds a promise for creating value for owners and facilities management organizations in various applications, including locating components, facilitating real-time data access, checking maintainability and automatically creating digital assets. Real-time correspondence of the model with an actual facility has not been studied. Hence, BIM appears to create a promising platform if equipped with sensing technology which can facilitate real-time update of the facility.

RFID plays a major role in facilities management, such as in security, inventory, construction site delivery logistics and materials tracking, document tracking, product life cycle tracking and building energy controls (Jaselskis and El-Misalami 2003, Wing 2006, Kelm et al. 2013). Automating the tagging and tracking assets using RFID can increase the value of FM (McAndrew et al. 2005). RFID tags are able to read multiple tags simultaneously and uniquely recognize facility items, store information regarding maintenance history of these items, and continuously update the information in real-time (Ergen et al. 2007a).

\section{Indoor localization}

Indoor localization refers to locating an object or person in an indoor environment by the use of context-aware information. While the Global Positioning System (GPS) has become synonymous with outdoor location tracking and navigation, there have been attempts with various sensing technologies for localization in an indoor environment. Sensors of different types have been tested and proven in the outdoor construction industry. GPS technology, uses trilateration from multiple satellites, provides context-aware information and does not require any pre-installed infrastructure for location determination (Pradhananga and Teizer 2015, Vasenev et al. 2014, Behzadan et al. 2008). Trilateration is one of the basic principles of location estimation, in which it uses three or more data points - with additional information such as signal strength - to determine relative position of an object. Trilateration is commonly used in GPS navigation, tracking cell-phones via towers and location of an earthquake epicenter (Anumba and Aziz 2006). Similarly, Ultra Wideband (UWB), a wireless radio frequency based on a real-time locating system (RTLS), also provides context-aware information that can be implemented to track and determine the location of resources in a jobsite (Teizer et al. 2007, Teizer et al. 2008,
Cheng et al. 2011). The UWB utilizes multiple readers to identify the location of tags, but it requires careful installation of these readers at known locations. And although both GPS and UWB provide high precision locations, both of these technologies possess several drawbacks for use indoors (Teizer et al. 2008, Cheng et al. 2011, Khoury and Kamat 2009). UWB and Indoor GPS both require due to multi-path effects a line-of-sight between the receiver and transmitter or reference tag, respectively and require considerable time for deployment. Moreover, GPS cannot be used in an indoor environment (Ogaja 2011), and neither technology is able to record the timestamps which is vital for localization. Cameras and vision technology have been used in construction for project monitoring and safety (Bohn and Teizer 2010, Memarzadeh et al. 2012, Yang et al. 2014, Yang et al. 2011, Yang et al. 2010, Teizer and Vela 2009), but have not been integrated with BIM based FM. Vision based technologies are not suitable for tracking objects because they do not (yet) rely on unique identification for each utility, and therefore cannot distinguish between similar objects (Kropp et al. 2014).

Liu et al. (2010) proposed an indoor localization and visualization environment using $2 \mathrm{D}$ laser scanner and inertial measurement units (IMU). A human-operated backpack system and image based rendering was used to process location. Bernoulli et al. (2010) also utilizes an IMU and an algorithm to map indoor travel to a simple computer aided design (CAD) model. However, IMUbased localization suffers from drift in the sensors (Taneja et al. 2012). Additionally, these systems require large computing time and have yet to be visualized real-time in a BIM model.

Indoor localization using RFID can be used to pin-point the current position of the reader and lead the worker with the RFID reader to the correct utility (Pradhan et al. 2009). An approach to localization using an array of passive RFID tags placed on the floor of an experimental testbed has been implemented by Park et al. (2009) for indoor mobile robot movement. Signal strength control was used for improving performance of position estimation. However, many problems resulted in high error when using RFID's received signal strength (RSSI) alone. The RSSI values do not correspond to physical positions and can change with various environmental conditions (Choi et al. 2009, Fink and Beikirch 2011, Wang 2011). Since RFID technology, alone, cannot pinpoint a location, the technology requires additional algorithms or particle filters to estimate the location.

Simultaneous localization and mapping (SLAM) is a popular technique used for autonomous vehicles to use sensing technology to build a map within an unknown environment (without a priori knowledge) while simultaneously using the map to compute its location. There are various forms of SLAM implemented in different 
environments, but the basic formula and structure are the same. The basic parts of SLAM include landmark extraction, data association, state estimation, state update and landmark update. Durrant-Whyte and Bailey (2006) use the recursive Bayesian formulation for the real-time probabilistic estimation of location at a time instant $k$. A SLAM algorithm has also been evaluated for 2D trajectory tracking using passive RFID tags (Yang et al. 2010). The work by Bekkali et al. (2007) introduced a new positioning algorithm using two mobile RFID readers and passive or active RFID tags as randomly distributed known landmarks. However, algorithms that require a large number of RSS measure samples to achieve good accuracy can be a limiting factor in processing and storage capacity. Haehnel et al. (2004) integrate indoor tag locations and laser scan data to produce accurate maps of RFID tags, which can be used for accurate localization of the robot and for moving objects without odometer information.

Current techniques to determine the location of a passive RFID tag require additional labor-intensive enhancements, such as sensor histogram models or pre-mapping of tags. Therefore, to eliminate the need for these additional enhancements, Deyle et al. (2008) developed a system that utilizes a robot that houses a physical sensor model and a particle filter that utilizes direct-path and multi-path RF propagation models, which are integrated into the filter by low variance resampling that predicts the far-field read range. Bouet and dos Santos (2008) surveys the current state-of-art of RFID localization techniques and concludes that the choice of technique and RFID technology significantly affects the granularity and accuracy of the location information but also the whole cost and the efficiency of the RFID system. Therefore, the best suited indoor localization technique depends on the final application.

RFID technology is shown to be effective in applications for emergency response and search and rescue (Ergen et al. 2011). Chen and Peña-Mora (2012) use RFID tags for building assessment in the context of disaster occurrences and show that the tags allow an economic and effective way to store and track geographically distributed information relevant to urban search and rescue (US\&R). Rüppel and Stübbe (2008) proposed an indoor navigation system that utilizes RFID, ultra-wide-band (UWB) and a wireless local area network (WLAN) for emergency response and recovery. However, testing has not been conducted to evaluate the accuracy and reliability of the system. Unfortunately, the previous systems rely heavily on the infrastructure of a facility, such as power or network connections. This presents a problem in the event of an emergency, which may result in a power outage or network failure. If the infrastructure goes down, so does the technology relying on it. Therefore, there is a need for a self-sufficient technology that does not require continuously running technological infrastructure directly from the facility.

Radio Frequency Identification (RFID) technology offers a solution because it can be self-reliant. Passive tags do not require power to activate them and a mobile reader can be battery powered (Costin et al. 2013, Goodrum et al. 2006). Additionally, prior knowledge of the layout of a facility can improve the performance of RFID localization (Taneja et al. 2012, Ergen et al. 2007b). Therefore, integrating BIM with RFID technology would assist in localization and navigation of a facility even in a power outage.

\section{Goals and significance of passive RFID and BIM- enabled location tracking}

The scope of this research is the use of passive radio frequency identification (RFID) as the sensing technology and focuses on the operation and maintenance phase of the lifecycle of an office building. It is assumed that objects in the facility are already tagged with RFID tags. Ideally the objects would be tagged via supply chain management, but the developed approach will also extend to the additional tagging of objects already in place inside a facility. The objectives of this paper are (1) develop a framework that utilizes the integration of commercially-available RFID and a building information model; (2) evaluate the framework for real-time resource location tracking within an indoor environment; and (3) develop an algorithm for real-time localization and visualization in a BIM. The goal is to achieve a system accuracy within $3 \mathrm{~m}$ at $95 \%$ precision, as defined by the study of Taneja et al. (2012). This means that real-time visualization display must be within $3 \mathrm{~m}$ of the actual location $95 \%$ of the time. An accuracy of $3 \mathrm{~m}$ is small enough to guide personnel to the general location of components or equipment in a facility, which then the personnel can distinguish the desired item. Previous works of this research (Costin and Teizer 2014) have demonstrated the ability to achieve this accuracy in a controlled test environment, and the next step is to deploy on a larger scale. This paper only deals with passive RFID location tracking based on utilities located inside a facility. The position of the reader can be visualized in realtime in the BIM model, which will aid in the determination of current location within the facility, as well as facilitating navigation

The contribution of this paper is to demonstrate the importance of utilizing a BIM model to increase the accuracy of indoor localization. This paper presents the fusion of commercially available passive RFID technology, indoor localization techniques and a BIM model for effective real-time indoor location tracking and visualization, without relying on existing building or wireless infrastructure. 


\section{Methods}

\section{Hardware and equipment}

The success of this research lies with the ability to link commercially available RFID tracking technology and a BIM. Ideally, the RFID tags utilized in this research are from the initial construction of the building and installment of the objects from supply-chain management, thus reducing the amount of tags needed to be installed at a later date. After the completion of the construction phase of the facility, all data and information (drawings, database, model, etc.) are turned over to the owner in order to keep up with operation and maintenance of the building. Any resource that contains an RFID tag can be read essentially anywhere in the facility (within range of the readers) and be shown in the BIM model in realtime. The facilities management utilizes the stored tag location of the utilities in order for facilities management, including real-time feed of surround tagged objects, automated scheduling for maintenance, automated summary and reporting and indoor localization (Costin et al. 2012a).

The same prototype mobile cart throughout this research was used. The mobile cart (see Fig. 3) comprises of one ThingMagic M6 UHF RFID reader that connects to four MTI MT-262006/TRH/A 902-928 MHz circular polarized antennas placed on the top, front, left side and right side. The M6 uses WiFi to transmit the data reads to a computer for storage. Since the cart mimics ones used by FM, there is space below to hold the maintenance tools and equipment.

Additionally, the cart contains an OceanServer OS5000 precision 3-Axis Tilt Compensated compass that was used for both ground truth data and supplemental data for the location algorithm. The features of the 1 "x1" size compass include 3 axis magnetic sensors, 3 axis accelerometers and 50 MIPS processor that provide precise tilt compensated heading, pitch and roll information. The compass accuracy is $0.5^{\circ}$ root mean square (RMS) while level, $1^{\circ} \mathrm{RMS}< \pm 30^{\circ}$ tilt, and $1.5^{\circ} \mathrm{RMS}< \pm 60^{\circ}$ tilt. Although the compass is accurate enough to provide the location path information, the goal of this research is to utilize the solely RFID and BIM integration data to estimate the tracking information. However, some data, e.g., cardinal direction, from the compass will be used to supplement the RFID-BIM integration data.

Between active and passive RFID, passive tags are shown to be the most suitable for an indoor application of tracking workers, materials and equipment due to their small size, low cost and low maintenance requirements (Costin et al. 2012a). Passive tags also hold potential benefits for the operation and maintenance phase of a facility. First, passive tags are inexpensive (US\$0.10 each or less) and can essentially last for a long time in typical indoor environments. Moreover, a facility can potentially have hundreds or thousands of tag objects, adding unnecessary costs. Although the passive tags have a significant smaller read range than an active tag, it is not necessarily beneficial to have a long read range in the first place. Having a long read range means more tags will be read at a certain time, potentially causing data overloads (Costin et al. 2012b). Missed reads (false positives) can also result due to the high volume of tags. One limitation to passive tags is the fact that they are easily obstructed by objects. In this case, active or semi-active tags could be used.

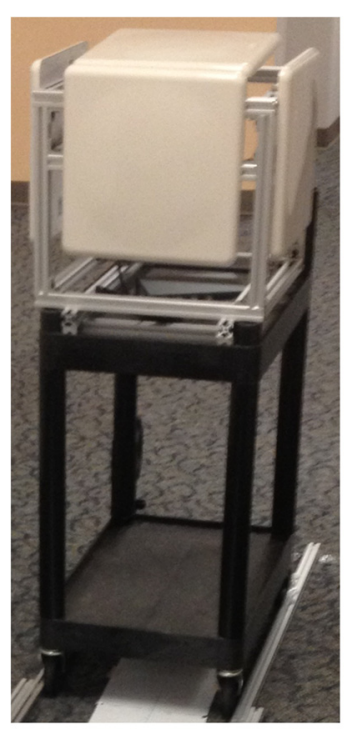

(a)

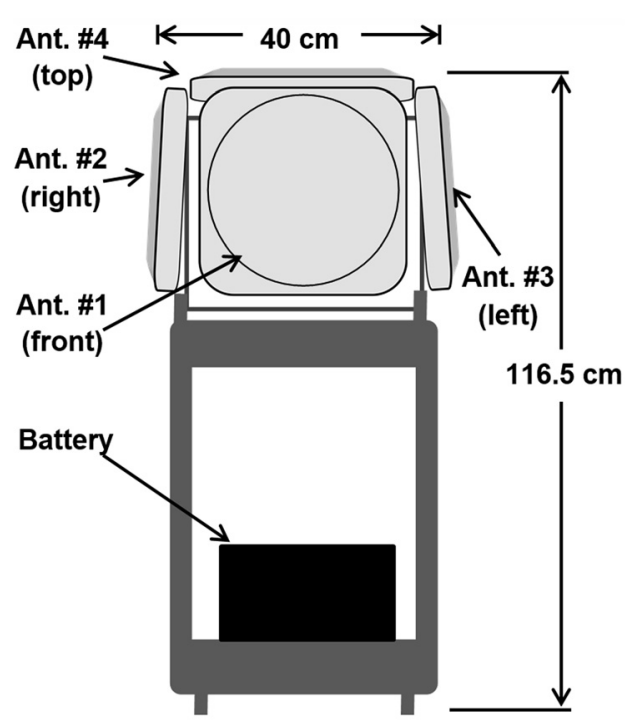

(b)

Fig. 3 a Mobile cart and (b) front view with dimensions 
Passive tags require nonvolatile memories that retain the values stored even when the device is not powered, which is typically less than 200 bytes (Ahson and Ilyas 2008). This means that any additional information about the tagged object (e.g. product name, maintenance history, warranty) must be stored in a separate database. There are also benefits to having data not directly stored on the tag. First, the more data the tag has, the more power it requires to send back, ultimately reducing the read range. Without the data being backed up in a database, all data would be lost in the event the tag is damaged, lost or destroyed. Having the data stored in the database allows for the programming of an additional tag with the same unique ID in order to link it with the data. Not storing the data in the tag is also desirable for security reasons. Bhaskar (2007) explains potential security concerns with RFID and the data store on the tags, including tag cloning, tag data corruption, data theft and illicit tracking and data mining. Fortunately, there are several security measures and techniques, e.g., encryptions, protocols, that are used to prevent security breaches. Finally, the database can hold substantially more data over the lifetime of the tagged object than on the tag itself. Moreover, if the tag were to be destroyed, the data stored on it could be lost if not stored elsewhere.

\section{Technology and software framework}

The framework presented in this paper allows for the use of any commercial BIM software and RFID technology. In this research, Tekla Structures 19.1 was selected as the BIM platform and Trimble ThingMagic for the RFID technology. The first step was to integrate RFID and BIM by means of the application programming interface (API) of the software and hardware components. Figure 4 shows the BIM and RFID framework in graphical form Costin et al. (2014). A prototype software application was developed in Visual C\# 2010 to be the central hub for communication between

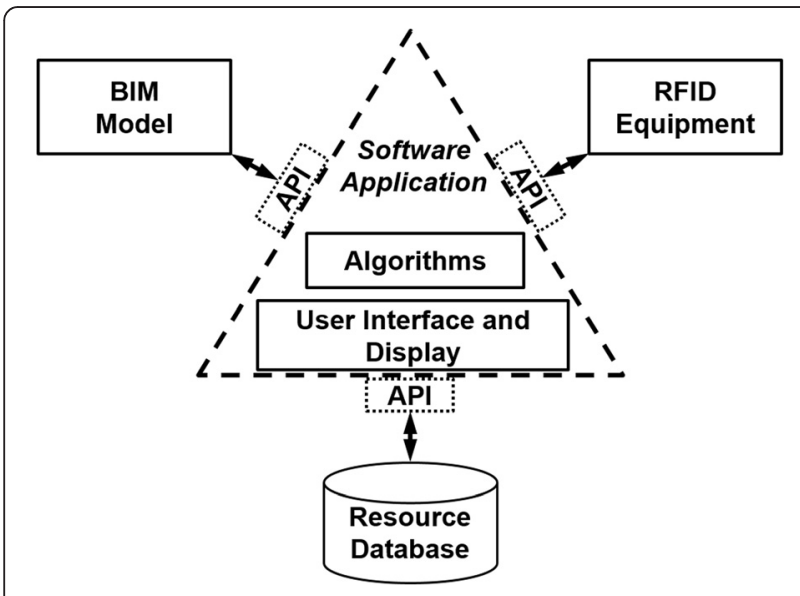

Fig. 4 BIM and RFID framework (Costin and Teizer 2014b) the software and hardware components. This application links communication between the RFID equipment, BIM model and resource database.

The software allows the data to be acquired: RFID tag ID, read frequencies, timestamp of read, received signal strength, antenna number and tag read count. The application also runs the location and visualization algorithms that locates and displays objects in the user interface for real-time visualization. In addition to storing all the geometric information (the standard 3D model), a BIM also has the capability of storing all the attributes for each of its elements, such as part number, material type, histories and scheduling, which have benefits for FM. An additional database has been implemented to store all the relevant data from the model that has been retrieved prior to running the algorithm. Retrieving the relevant data at first, rather than iterating through the BIM model each time the algorithm is run, will save substantial computing time since the BIM can be quite large in the amount of data it contains. Please note that computing time is not within the scope of this study. Additionally, each tagged object in the BIM is linked to a unique RFID tag ID, which is also stored in the database. For instance, whenever a tag is read, the corresponding object ID in the model is retrieved from the database (along with basic information about that object) and is displayed on the user interface. If additional information about that object is desired, then clicking on the tag ID will query additional information from the database, such as user manuals, warranties and safety specifications.

Previous publications of this research used a synchronous planned read method for capturing the RFID data. This means for a starting time, $\mathbf{k}$, the reader would use a read cycle to capture all the tag reads and then return an array of the tag read data. As stated from (Trimble 2014):

"It performs the operation synchronously, and then returns an array of TagReadData objects resulting from the search. If no tags were found then the array will be empty; this is not an error condition. When performing a synchronous read() operation the tags being read are buffered on the reader and stored in the reader's Tag Buffer. During a single read() operation tag deduplication will occur on the reader so re-reads of the same tag will result in the tag's ReadCount metadata field to be incremented, a new TagReadData instance will not be created for each. The reader specific hardware guide should be referenced for information on the size of the Tag Buffer."

A synchronously read cycle is like the following example for one reader with 4 antennas: 1 ) read tags using antenna \#1 and store data in a queue; 2) read tags using antenna 
\#2 store data in a queue; 3) read tags using antenna \#3 store data in a queue; 4) read tags using antenna \#4 store data in a queue; and 5) write (send) all queued data to computer for processing and storage. The cycle repeats only after duration of the previous loop is complete. In other words, the data from the tag read cannot be processed until all the readers complete its duration, i.e. the each reader in the system is not reading continuously. There are two primary Gen2 settings that control readerto-tag communications; Tari and link rate. Tari is duration of the reader's signal for reading tags, and the link rate is the duration between each signal reading. For example, reader " $\mathrm{A}$ " turns on and reads for $2 \mathrm{~s}$ and then turns off for $1 \mathrm{~s}$. In this case, Tari is $2 \mathrm{~s}$ and the link rate is $1 \mathrm{~s}$, and the duration of the cycle is $3 \mathrm{~s}$. There is a maximum speed (i.e. amount of data per second) at which the tags can be read, and it is limited by the length of the Tari and link rate. By default of the ThingMagic reader, if a smaller Tari is selected, the link rate is automatically increased. Tari values offered are 6.25, 12.5 and $25 \mu \mathrm{sec}$, which are automatically paired with link rates of 40, 80 and $160 \mathrm{kbps}$ by the ThingMagic reader (Trimble 2014). Thus, the longer the Tari the more information that can be picked up at during that read. However, on the downside, the longer the Tari, the longer the overall read cycle which can negatively affect other readers in a multi-reader system. Only one reader can read at a time (other readers are dormant until their turn in the loop), resulting in missed reads due to other readers not being able to read concurrently (this paper only deals with one reader with four antennas, in which the variation of Tari and link rate durations are negligible).

The RFID technology was configured to read continuously, using an asynchronous reading method with event listeners (callbacks). As stated from (Trimble 2014):

"It returns immediately to the calling thread and begins a sequence of reads or a continuous read, depending on the reader, in a separate thread... The results of each read is passed to the application via the ReadListener interface; each listener registered with the addReadListener() method is called with a TagReadData object for each read that has occurred. In the event of an error during these reads, the ReadExceptionListener interface is used, and each listener registered with the addReadExceptionListener() method is called with a ReaderException argument."

Unlike a synchronous read plan, which is a loop, an asynchronous read plan uses a read listener that stores tag information as soon as a read object occurs. In other words, the computer is always "listening" for tag reads, and once a reader picks up a tag read, the tag data is immediately stored, i.e., each reader is continuously reading. Note that, although the readers are reading continuously, there may be a lag in data storage if multiple readers are reading simultaneously, but the time of the lag is negligible. There are multiple benefits of using asynchronous reading including utilizing multiple readers/antennas simultaneously, reading multiple tags per reader/ antenna, and not having to miss tags from readers/antennas being dormant.

\section{Localization and visualization algorithm}

One major contribution of this research is localization of a user (FM) in a facility in real-time, and displaying the user's position directly in the BIM model (similar to GPS display). The current algorithm has been updated and modified from previous works of this research (Costin et al. 2013, Costin and Teizer 2014, Costin et al. 2014). This algorithm is modified to integrate RFID data from a mobile reader as seen in Fig. 3, which is referred to as "cart". Fig. 5 is the current, modified process flow diagram of the localization and visualization algorithm. What distinguishes this from previous versions are (1) it incorporates the BIM model data directly, and (2) the "Calculate Current Position" step was changed from a simple mean location technique to more complex multilateration techniques, as described in more detail later.

The algorithm works by first by extracting data from the building information model at time: 0 . This process eliminates the time needed from searching the entire BIM model for corresponding information at runtime of the algorithm. The data extracted from the BIM model include all the stored RFID tags (landmarks), RFID tag coordinates and building information model object properties, which are then stored in the resource database. Since each tag is assigned to the object it is tagged on, all the object properties from the building information model are also extracted. This data will be included in the supplementary data. The inherited properties utilize by the algorithm include, but not limited to - since the algorithm can be enhanced -, the object's location, normal direction (cardinal direction normal to its outer facing side), and relative objects and properties (e.g. what objects are surrounding it). The extraction only needs to happen once at the beginning of the algorithm, since all the data can be stored in the database. Although it is not in the scope of this paper, it is conjectured that querying the data in a database takes less computational time that querying through a model. This claim is based on laboratory testing and still needs to be scientifically validated.

Then, for each instance at time event $\mathbf{k}$ (rounded to the nearest second for this research), the algorithm receives the tag read data from the RFID system. After the reader sends the signal, a list of the tag data is returned to the computer. Concurrently, for each tag read, the 


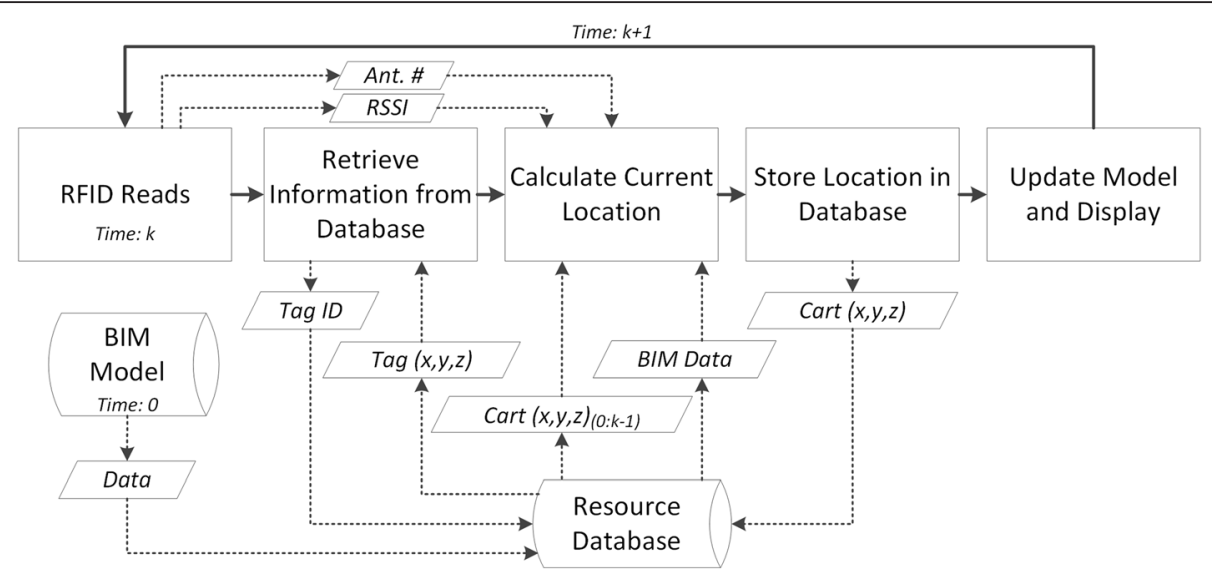

Fig. 5 Process flow of localization and visualization algorithm (modified from Costin et al. 2013)

$(\mathrm{x}, \mathrm{y}, \mathrm{z})$ location and supplemental BIM information of that object is retrieved from the database. Using all the tag locations (tag ID) at time event $\mathbf{k}$, the algorithm firsts computes the cardinal direction the cart is facing. It then uses a version of multilateration to compute the carts location. Multilateration uses three or more data points (with additional information) to determine relative position of an object (e.g. GPS). Since the locations of the tags are relatively close to the reader, environmental factors (e.g. curvature of the earth, atmosphere, temperature, etc.) can be neglected.

In additional to the supplemental BIM data, additional information is used to adjust each location estimation. The received signal strength indicator (RSSI) paired with the antenna of each tag read are also used to provide the location of the tag relative to the reader (Eq. 1). For example, if antenna \#3 reads a tag at high RSSI, the tag has high probability it will be on the left side of the cart. Additionally, if antenna \#3 reads a tag with low RSSI, the tag may be further away from the cart or even result from multipath. There are distance factors, based on testing (see Power output and received signal strength), which are placed depending on the RSSI value. These factors give high RSSI values higher probability of being closer to reader. Next, the algorithm uses previous locations to estimate the probability of the current location (Eq. 2). For instance using certain thresholds (e.g. time and location), it would nearly be impossible to be at one

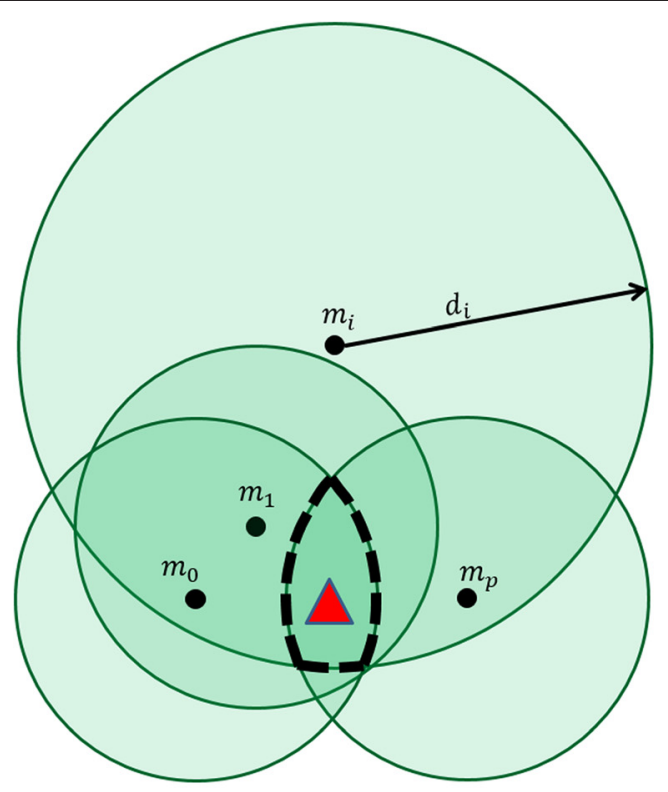

(a)

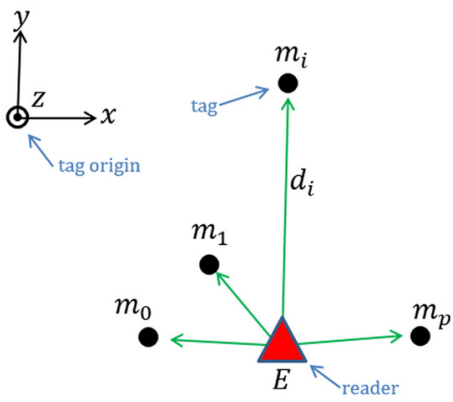

(b)

Fig. 6 Multilateration: (a) intersection and (b) distance equation 
location and jump to another location in a minimal amount of time. This can be seen in the jaggedness of the paths in Figs. 12a, 13a and 14a of the results section, which have not incorporated this portion of the algorithm. The updated location estimation is then stored in the database.

Finally, the calculated location is then sent to the BIM model, and the updated location is displayed on the user interface at a given refresh rate. A refresh rate is the number of times the display is updated per second $(\mathrm{Hz})$. The algorithm repeats at time $\mathbf{k}+\mathbf{1}$. It is important to note that $\mathbf{k}$ can be any duration and, thus, $\mathbf{k}+\mathbf{1}$ signifies the next event, and not necessarily "time + plus $1 \mathrm{s."}$ Therefore, during runtime, the visual display updates the current position of the cart in the model (similar to how GPS monitor displays the car while driving). So as the cart is being pushed through the facility, the corresponding movements are represented in real-time on the display, giving the user a $3 \mathrm{D}$ view of the current location relative to the model.

In this paper, two approaches for multilateration are used; (1) intersection of tags and (2) the distance formula. The intersection approach uses geometric circles produced by the tags (Fig. 6a) while the distance equation approach uses the distances between the reader and each tag location read (Fig. 6b). These approaches are implemented under the "Calculate Current Location" process in Fig. 5, and below describes how they are used for the BIM-RFID integration.

Both approaches aim to find the location vector, $\vec{E}$, of the mobile cart. The vector is defined as:

$$
\vec{E}=(\boldsymbol{x}, \boldsymbol{y}, \boldsymbol{z})
$$

Since the height of the cart is known, $\mathrm{z}$ will be constant. Therefore, the $\mathrm{x}$ and $\mathrm{y}$ coordinate will need to be determined at each iteration.

For each tag read data, each tag location vector, $\overrightarrow{\boldsymbol{m}}$, from the model is used.

$$
\overrightarrow{\boldsymbol{m}}_{0}, \overrightarrow{\boldsymbol{m}}_{1}, \ldots, \overrightarrow{\boldsymbol{m}}_{i}, \ldots, \overrightarrow{\boldsymbol{m}}_{p} \text { where } 0 \leq i \leq p \text { and } \overrightarrow{\boldsymbol{m}}_{i}=\left(x_{i}, y_{i}, z_{i}\right)
$$

The location vectors are known and will be obtained from the model. The relationship between RSSI and distance can be shown in the following equation (Wang et al. 2009):

$$
\text { RSSI }[\mathrm{dBm}]=-\left(10 * n * \log _{10}(d)+A\right)
$$

Where $\boldsymbol{A}$ is the absolute RSSI $1 \mathrm{~m}$ away from the reader and $\boldsymbol{n}$ is the signal propagation coefficient. Both parameters must be determined empirically (this is shown in Section 5.3). Using the RSSI from each tag

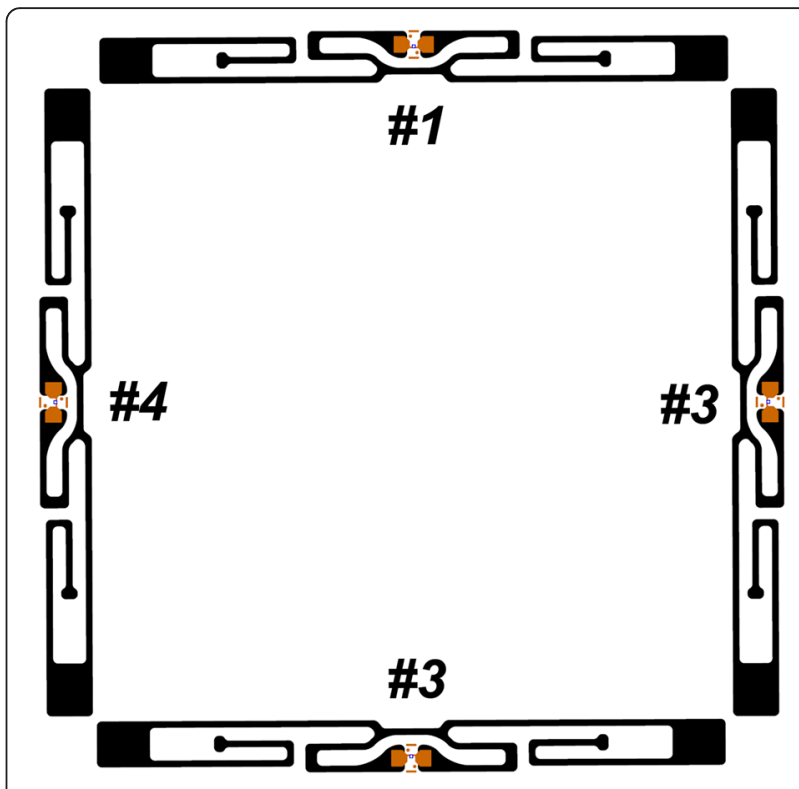

Fig. 7 Four different RFID tag orientations

read data, Eq. 3 can then be solved for $\boldsymbol{d}$, which is used in both multilateration approaches.

Each iteration of reading produces a list of tag read data. How this list is processed for localization is what differentiates the intersection approach from the distance equation approach. The intersection approach uses geometric circles produced by the tags (Fig. 6a). The center of the circle is the known coordinate of the tag, and the radius is the RSSI that is calculated by Eq. (3). Given each tag read radius, $\boldsymbol{d}$ (calculated from the RSSI), let $\boldsymbol{C}$ be the corresponding circle and $A \boldsymbol{c}$ be the area of intersecting circles. Then, $A \boldsymbol{c}$ is calculated by taking the union of all $\boldsymbol{C}$ in each tag read data (Eq. 4).

$$
A c=C_{0} \cap C_{1} \cap \ldots C_{i} \cap \ldots C_{p}
$$

If the circles do not overlap, the radii of each $C$ are iteratively increased by a given weight (e.g. $.001 \mathrm{~m})$ until they do overlap. Given that complex geometries can be produced for $\boldsymbol{A c}$, the centroid of the area can be found by using the center of mass of the point cluster. The centroid coordinate is $\overrightarrow{\boldsymbol{E}}$.

The distance equation approach uses the distances between the reader, $\overrightarrow{\boldsymbol{E}}$ and each tag vector, $\overrightarrow{\boldsymbol{m}}$ (see Fig. $6 \mathrm{~b}$ ).

Table 1 Read range distances (cm) with respect to tag orientation

\begin{tabular}{llllllll}
\hline Tag Orientation & \multicolumn{2}{l}{ Trial } & \multicolumn{6}{ll}{} & Average \\
\cline { 2 - 6 } & 1 & 2 & 3 & 4 & 5 & 6 & \\
\hline$\# 1$ & 222 & 216 & 211 & 224 & 229 & 224 & 221 \\
$\# 2$ & 406 & 406 & 418 & 425 & 430 & 416 & 417 \\
$\# 3$ & 298 & 315 & 319 & 316 & 316 & 319 & 314 \\
$\# 4$ & 408 & 372 & 405 & 408 & 370 & 402 & 394 \\
\hline
\end{tabular}




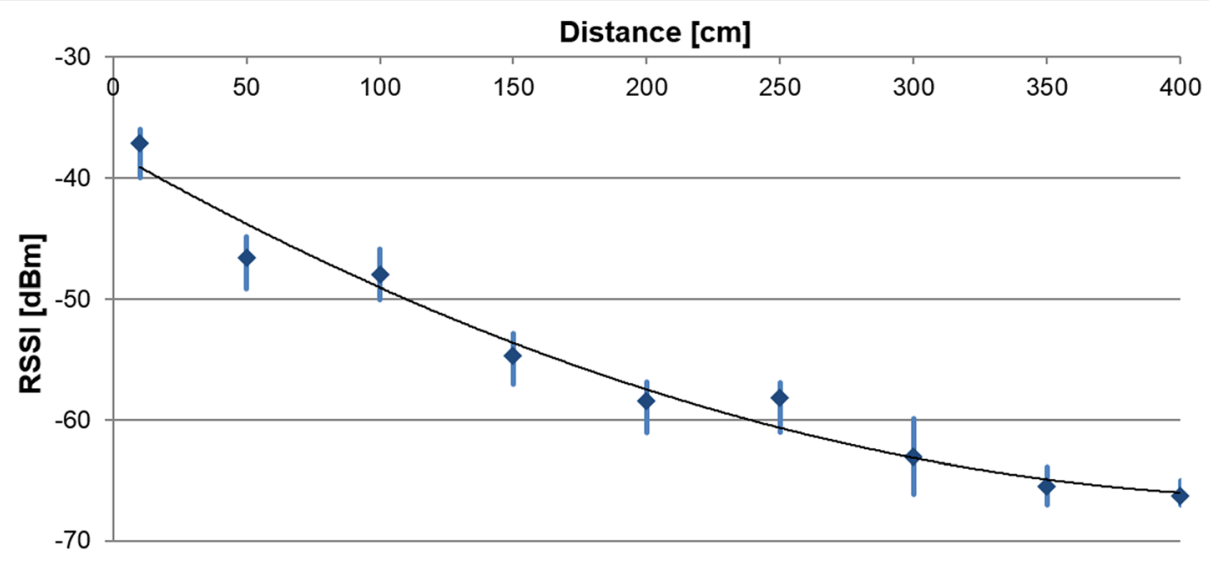

Fig. 8 RSSI with respect to distance

Each distance between the tag and the reader, $\boldsymbol{d}$, is formulated by Eq. 5:

$$
\boldsymbol{d}_{\boldsymbol{i}}=\left|\overrightarrow{\boldsymbol{m}}_{\boldsymbol{i}}-\vec{E}\right|=\sqrt{\left(\boldsymbol{x}_{\boldsymbol{i}}-\boldsymbol{x}\right)^{2}+\left(\boldsymbol{y}_{\boldsymbol{i}}-\boldsymbol{y}\right)^{2}+\left(\boldsymbol{z}_{\boldsymbol{i}}-\boldsymbol{z}\right)^{2}}
$$

Finally, knowing $\boldsymbol{d}$ (computed using RSSI and antenna number) a system of nonlinear equations for each computed $\boldsymbol{d}$ can be solved to find $\overrightarrow{\boldsymbol{E}}$. It is important to note that computing a system of nonlinear sometimes fails to converge to a solution. Therefore it is important to have a numerical computing environment, such as MATLAB, to run the computation and achieve an estimated result.

Both approaches may produce a path that is jagged, which is caused by error in the estimation. To smooth the path, a damper is placed in the algorithm, that uses the $(0: \mathbf{k}-\mathbf{1})$ cart locations to put limits on the kth time location. (Note from previous definition that $\mathrm{k}-1$ is the previous time instant and not a literal subtraction of time minus 1). For instance, the kth position would not be further away then what is physically possible. Therefore, $(0: \mathbf{k}-\mathbf{1})$ locations can estimate the speed, $\boldsymbol{v}$, of the

Table 2 RSSI (dBm) with respect to distance (Costin et al. 2012a, 2014a)

\begin{tabular}{llllc}
\hline Distance $(\mathrm{cm})$ & Max & Min & Avg. & Standard deviation \\
\hline 10 & -36 & -40 & -37.13 & 0.91 \\
50 & -45 & -49 & -46.66 & 1.42 \\
100 & -46 & -50 & -48.00 & 1.31 \\
150 & -53 & -57 & -54.72 & 1.40 \\
200 & -57 & -61 & -58.43 & 1.16 \\
250 & -57 & -61 & -58.17 & 1.30 \\
300 & -60 & -66 & -63.05 & 1.86 \\
350 & -64 & -67 & -65.53 & 1.07 \\
400 & -65 & -67 & -66.26 & 0.49 \\
\hline
\end{tabular}

cart and then set an upper bound for maximum distance for location at time instance k (Eq. 6).

$$
\nu_{\mathbf{k}}=\frac{\operatorname{distance}_{(0: \mathbf{k}-\mathbf{1})}}{\operatorname{time}_{(\mathbf{0}: \mathbf{k}-\mathbf{1})}}
$$

The number of time instances $\mathbf{k}$ that are the used in the speed equation can be determined by the user. Once the speed is determined, it can be used to as the maximum distance that the next $\mathbf{k}$ instance can be. Let $\max D$ be the maximum distance that can be traveled based on the speed calculated from the previous locations (Eq. 7), and $\boldsymbol{d c}$ denote the distance from the new estimated location at $\mathbf{k}$ from (0:k-1) locations (Eq. 8).

$$
\begin{aligned}
& \max _{k}=v_{(0: \mathrm{k}-1)} * t_{(0: \mathrm{k}-1)} \\
& d c_{k}=\sqrt{\left(x_{k}-x_{k-1}\right)^{2}+\left(y_{k}-y_{k-1}\right)^{2}} \\
& \text { if } d c_{k}>\max D_{k} \text { then } d c_{k}=\max D_{k}
\end{aligned}
$$

Therefore, if $\boldsymbol{d} \boldsymbol{c}$ exceeds $\max \boldsymbol{D}$ then the $(\mathrm{x}, \mathrm{y})$ of the estimate location at $\mathrm{k}$ needs to be adjusted (Eq. 9).

\section{Experiments and results to RFID technology performance}

Each manufacturer of sensing equipment (e.g. RFID tags, readers, antenna, etc.) gives a range of specifications. For example, a reader angle may say " $65^{\circ}$ plus or minus a degree." Although the range may not seem large, it still may lead to false results if improperly calculated. Therefore, it is proper to test the specifications of each newly acquired piece of equipment to verify it is in accordance with the manufacture's specification. Each unit varies slightly, and it is important to use what has been verified from the testing to produce proper results in the final system. This section provides the experiments and results used in this research. 


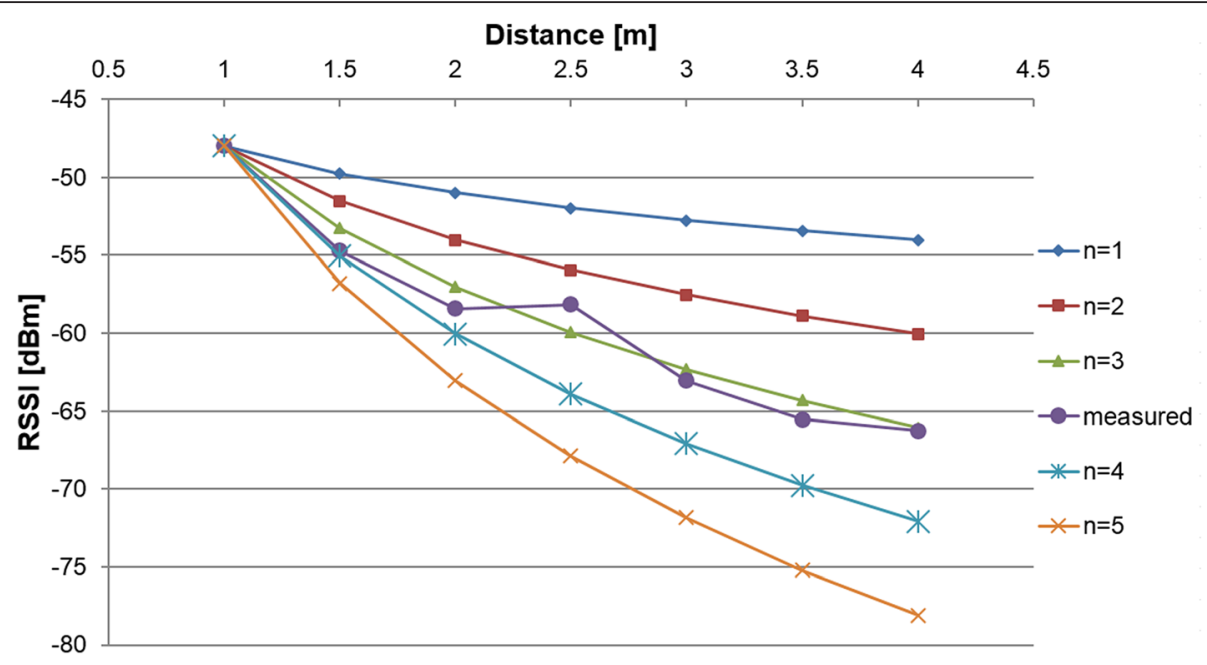

Fig. 9 Measured RSSI vs. calculated RSSI with different propagation coefficients

\section{Tag orientation}

Due to the properties of radio frequency waves, the orientation of a passive tag greatly affects the amount of power absorbed and reflected back to the reader, thus affecting the read range (Ahson and Ilyas 2008). The objective of this experiment is to determine the highest performing tag orientation. An open area with no obstacles was used to perform the test. A passive tag was attached to cardboard (to avoid interference) on a cart. The center of the tag and the center of the antenna are the same height. The cart was slowly pushed towards the reader's antenna (powered to $27 \mathrm{dBm}$ ) in a straight line. Once the reader indicated the tag had been read, the person pushing the cart stopped walking and marked the spot where he/she had stopped. The distance from the reader was measured and recorded. This activity was repeated six times for each of the four orientations respective to the reader: top (\#1), right (\#2), bottom (\#3) and left (\#4) as displayed in Fig. 7.
Table 1 displays the results of the experiment. Significantly, tag position \#2 results in the longest average read range of $416.8 \mathrm{~cm}$, and tag position \#1 results in the shortest average read range of $221.0 \mathrm{~cm}$. This experiment confirms that the tag orientation does affect the tag read range. The experiments and field trial for this research therefore position the tag in this manner for maximum performance.

\section{Antenna view angle}

The objective of this experiment was to determine the antenna beamwidth, which is the angle at which the reader can no longer read a tag. This was to verify the manufacturer's specifications that states the typical beamwidth is $63^{\circ}$ (Trimble 2014). A passive tag was attached to cardboard (to avoid interference) on a cart. The center of the tag and the center of the antenna are the same height. The cart was pushed sideway and parallel to the wall (with the antenna always facing the

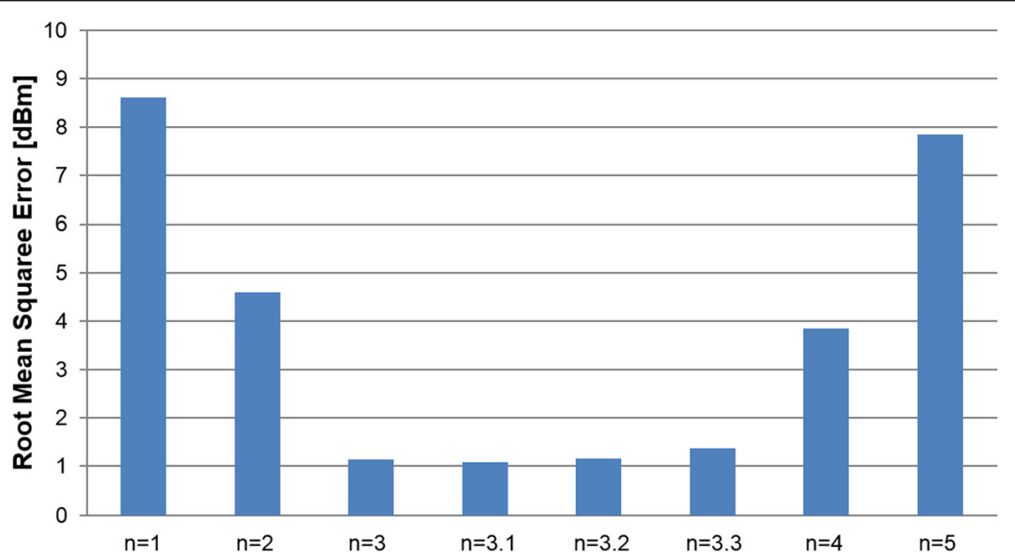

Fig. 10 Root mean square error between measured and theoretical RSSI at different propagation coefficients 
tag). First, the reader was recording the tag and was pushed to one side. When the tag stopped reading, the distance was recorded and the angle was calculated. Next, the reader was pushed to the other side until the tag was picked up. This was repeated for both sides numerous times. The maximum beamwidth was found to be $65^{\circ}$, which is very close to the specifications.

\section{Power output and received signal strength}

The RSSI, alone, cannot pinpoint the location, but can contribute data for the estimation of distance. Most received signal strength indicator (RSSI) localization methods operate the reader at a high output power. The ThingMagic M6 UHF RFID has a maximum output power of 31.5 $\mathrm{dBm}$ which allows for a maximum read distance of $9 \mathrm{~m}$. Additionally, the reader has a maximum tag read rate of 750 tags/s and operates at a frequency between 902$928 \mathrm{MHz}$. Using the highest allowable power results in receiving a large number of tag reads, large amounts of noise and backscatter. For indoor applications, it may not be most efficient to have the highest power output. Reducing the power output from the transmitter limits the range of the signal, ultimately reducing the amount of backscatter and noise and improving the accuracy of the system. In this research, a power output of $27 \mathrm{dBm}$ was chosen, which results in a maximum read range of $420 \mathrm{~cm}$.

This research conducted experiments to compare the distance of the tag with the RSSI values to help develop RSSI probability maps. More detailed information can be found in Costin and Teizer (2014). Figure 8 shows how the RSSI decreases with the increase of LoS distance, $d$. However, having a high RSSI does not necessarily mean the tag is far away. Other factors, such as tag pose or interfere, can result in a low RSSI. For instance, an RSSI of -41 will result in a distance of around $100 \mathrm{~cm}$, but an RSSI of -75 can lie within $100-200 \mathrm{~cm}$. Therefore, the higher the RSSI value, the higher the probability that the tag is close to the reader.

The average RSSI values from Table 2 are compared with the theoretical values from Eq. 3 to determine the propagation coefficient, $\boldsymbol{n}$. The absolute RSSI value, $A$, at $1 \mathrm{~m}$ is 48 . The values of $\boldsymbol{n}$ are varied from 1-5 and are shown in Fig. 9. Since the measured values are similar to $\boldsymbol{n}=3$, other values within .1 are measured. Figure 10 displays the root mean square error (RMSE) between theoretical and measured RSSI. Based on the findings, a propagation coefficient of 3.1 $($ RSME $=1.08)$ will be used in the algorithm. It is to note that these calculations should be carried out with the respected RFID equipment used.

\section{Technology and software framework performance}

Preliminary testing was conducted to validate the accuracy and reliability of the developed RFID-BIM framework. The first part of the experiment was to show that

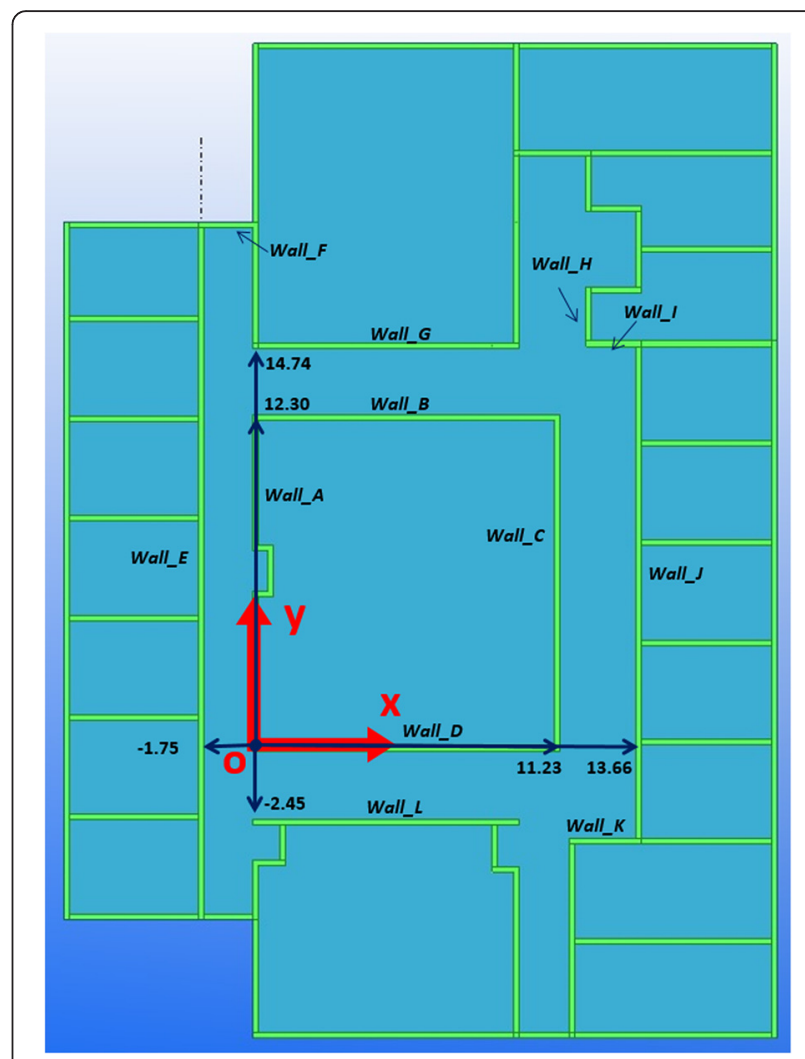

Fig. 11 Plan view of floor layout, dimensions [in meters]

the application can successfully link to the readers and database (Costin et al. 2012a). 20 unique tags were programmed to represent different utilities within a facility. Each utility is different, but similarities were made to see the application can distinguish the differences. For instance, two light bulbs are identical, except they are at different locations and have different installation dates. The application was deployed, and the tags were first placed one at a time to see if the correct information would be retrieved from the database and displayed properly on the screen. Once they all passed, a couple of tags at a time were placed in front to see if all their correct information would show. Finally, all tags were placed in front and all the correct information was displayed correctly.

Table 3 Comparison of updates at various times

\begin{tabular}{llcc}
\hline $\begin{array}{l}\text { Refresh } \\
\text { Rate }(\mathrm{Hz})\end{array}$ & $\begin{array}{l}\text { Seconds } \\
\text { per Refresh }\end{array}$ & $\begin{array}{l}\text { Average Number of } \\
\text { Reads per Refresh }\end{array}$ & $\begin{array}{c}\text { Average Distance }(\mathrm{m}) \\
\text { Covered per Refresh }\end{array}$ \\
\hline 1.00 & 1 & 1.9 & 0.54 \\
.50 & 2 & 3.2 & 1.07 \\
.33 & 3 & 4.9 & 1.61 \\
.25 & 4 & 6.3 & 2.15 \\
.20 & 5 & 7.7 & 2.69 \\
\hline
\end{tabular}




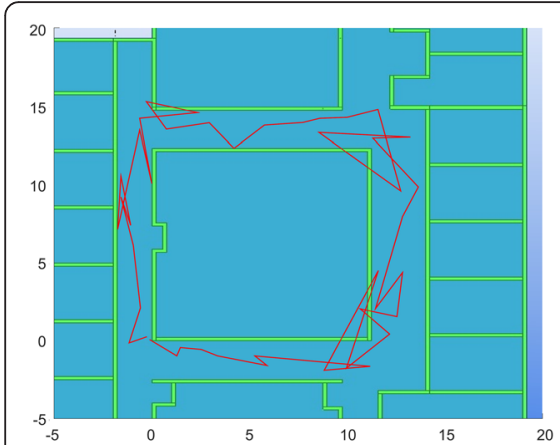

(a)

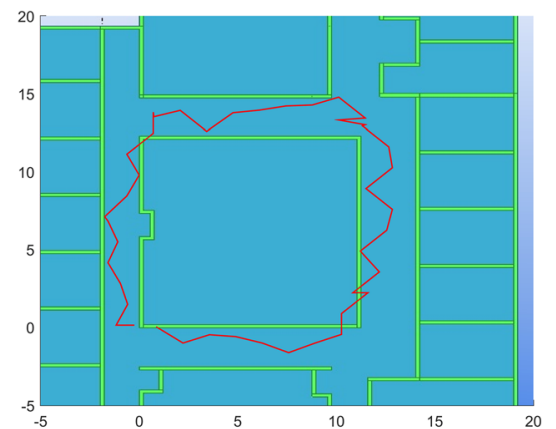

(b)

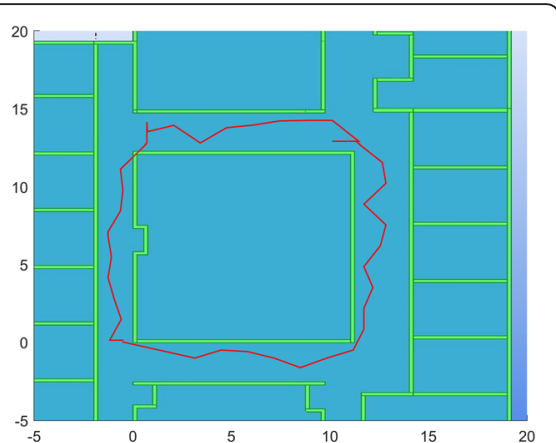

(c)

Fig. 12 a Original, raw data of the path of the average localization approach (without BIM), (b) data processing through filter to reduce jaggedness (with no BIM constraints), and (c) final algorithm: data processing through filter to reduce jaggedness with BIM constraints [in meters]

Thereafter, Costin et al. (2012a) validated the visualization in the BIM model. In order to test the connectivity of the application with Tekla Structures, objects were assigned to each of the tags along with the $(\mathrm{x}, \mathrm{y}, \mathrm{z})$ coordinate of the BIM object. Different trials were done storing and retrieving the objects corresponding to the tag. Again, one tag after the other was placed in front of the reader to test if the correct information was being displayed, as well as highlighting the corresponding object in the BIM model.

Costin et al. (2014) conducted preliminary tests for the location algorithm. The algorithm used was primitive, and did not include the supplemental BIM model. Error in position is calculated as a distance between the point where the cart is actually located and the location of the cart calculated by the algorithm in both the $\mathrm{X}$ and $\mathrm{Y}$ directions. The goal was to achieve an accuracy of $3 \mathrm{~m}$ at $95 \%$ precision. The mean error for the $\mathrm{Y}$ direction was $0.84 \mathrm{~m}$ with a standard deviation of $0.72 \mathrm{~m}$. The mean error for the $\mathrm{X}$ direction was $-0.26 \mathrm{~m}$ with a standard deviation of $0.45 \mathrm{~m}$. Using a standard Z-test, the $95 \%$ confidence interval for the system error is $0.84 \pm 1.41 \mathrm{~m}$, which lies within $3 \mathrm{~m}$ target.

\section{Field trial}

A field trial was conducted to test the updated research. An academic building was modeled in Tekla Structures. Figure 11 displays the plan view of the third floor with the relative dimensions and BIM information used in the algorithm. Throughout the corridors, 30 unique RFID tags were arbitrarily placed in order to keep the testing as random as possible and eliminate bias. They were placed to make sure all areas of the corridor would be covered, and leave no "gaps" or "dead zones" where the cart won't pick up any of the tags. Unlike the preliminary testing, the heights and locations of the tags were not kept constant. The corresponding tag $(\mathrm{x}, \mathrm{y}, \mathrm{z})$ locations were then modeled into the BIM model.

The origin from the model floor was used as the origin for the testing (see Fig. 11). The cart was pushed clockwise around the corridor loop, stating at point $(-0.88$,1.20). The cart was pushed north until it reached the center of the next corridor $(-0.88,13.52)$, rotated counterclockwise $90^{\circ}$ proceed west to the ne12xt corridor (12.44, 13.52). Again it rotated and proceeded south until point $(12.44,-1.21)$, where it then rotated again and finished back at the original starting point. The trial

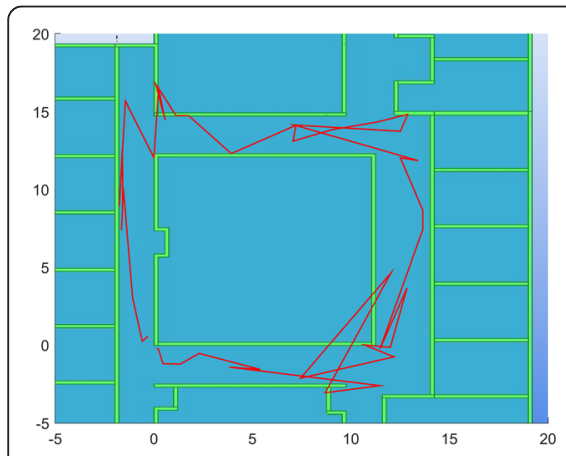

(a)

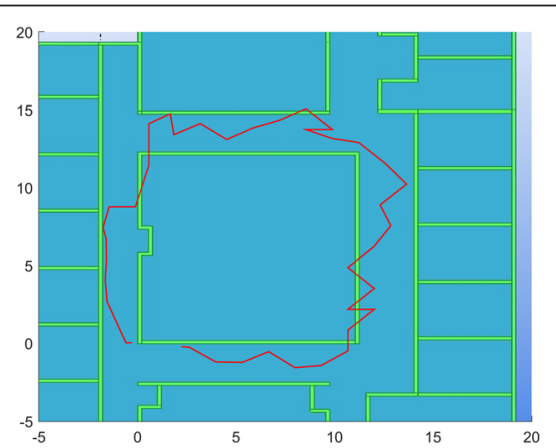

(b)

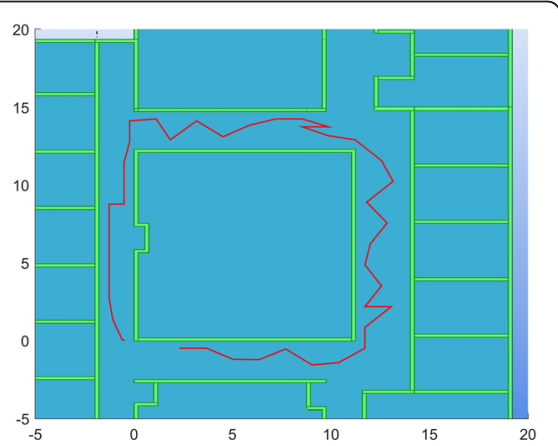

(c)

Fig. 13 a Original, raw data of the path of the intersecting approach (without BIM), (b) data processing through filter to reduce jaggedness (with no BIM constraints), and (c) final algorithm: data processing through filter to reduce jaggedness with BIM constraints [in meters] 


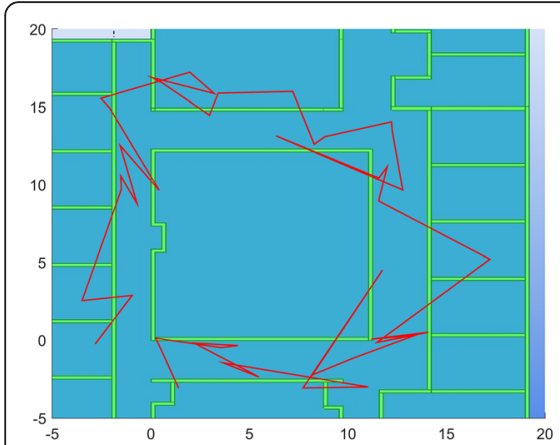

(a)

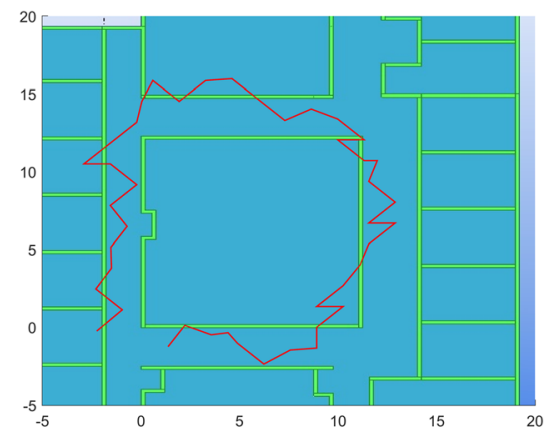

(b)

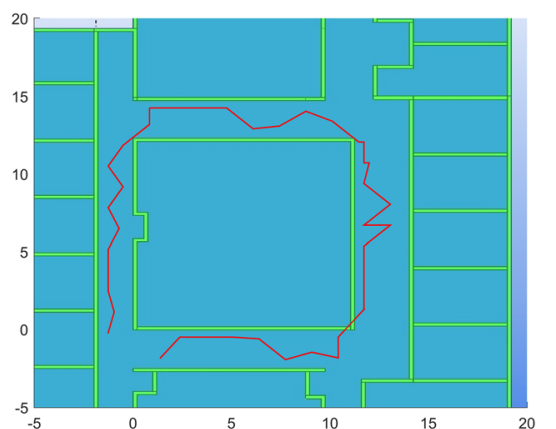

(c)

Fig. 14 a Original, raw data of the path of the distance equation approach (without BIM), (b) data processing through filter to reduce jaggedness (with no BIM constraints), and (c) final algorithm: data processing through filter to reduce jaggedness with BIM constraints [in meters]

was repeated twice more, and then repeated three more times going in the counterclockwise direction, for a total of 6 trials. The ground truth of the data was keep by keeping the time of each movement (e.g., start, stop, turn) and the compass data.

\section{Results}

\section{Display and location refresh rate}

Since the data is difficult to measure in real-time without additional performance measure (which are not in the scope of this paper), a suitable refresh rate needed to be chosen to update the location on the screen. For instance, a refresh rate $0.5 \mathrm{~Hz}$ would update the system every $2 \mathrm{~s}$. Table 3 compares times of refresh from 1-5 s, and Figs. 12, 13 and 14 display the corresponding paths (note that the paths are estimates strictly from the raw tag locations, and do not contain supplement information).

As the time to update the display increases, the smoother the path is and the more tags are read. However, on the flipside, the increase in time also increases the amount of distance covers, which could have more error in true location. Therefore it is important to have a balance. Based on the data from Table 3, a $0.50 \mathrm{~Hz}$ refresh rate was chosen because the average speed of the cart in the experiments was $.54 \mathrm{~m} / \mathrm{s}$, thus estimating a location about every $1.07 \mathrm{~m}$. On average, a $.50 \mathrm{~Hz}$ refresh rate collected 3.2 data reads from the RFID hardware.

\section{Cardinal direction}

The first portion of the algorithm estimates the cardinal direction that the cart is facing. This is key because knowing what direction the cart is facing makes the BIM

Table 4 Accuracy of cardinal direction estimates (RFID reads only) at $0.5 \mathrm{~Hz}$

\begin{tabular}{llll}
\hline & Straight & Turning & Total \\
\hline With unknown & $60 \%$ & $47 \%$ & $59 \%$ \\
Without unknown & $72 \%$ & $56 \%$ & $71 \%$ \\
\hline
\end{tabular}

model supplement information more reliable. Take walls, for example. If the cart is traveling north up a corridor, we know which wall is on the left side. Therefore, if the left antenna captures the read it has a higher weight placed on the location of the tag read, versus if the right antenna captured it from multipath. In all the trails, there were a total of 281 estimations at a refresh rate of $0.5 \mathrm{~Hz}$. If the algorithm didn't have enough information to estimate a cardinal direction, it would report out an "unknown" data point. The algorithm also didn't take into account, any previous estimated directions and so it is only estimating based on the current reads at the time of the estimation. Table 4 compares the accuracy of estimating the cart's cardinal direction by the tag reads only. It includes the percentage to true estimates with the "unknown" included, as well as without. Additionally, it separates out when the cart is on a straight path as well as turning.

Table 4 shows that algorithm can estimate a true cardinal direction correctly $72 \%$ of the times it has enough information to estimate when traveling straight. With only $47 \%$ accuracy, the algorithm has difficulties estimating the true direction when it is turning. This can be a result of two reasons: (1) it only calculates true directions, i.e. it does not do north-east, or (2) it may capture tags reads during turning which may interfere with the calculations. However, this research uses the actual data from the compass to determine the direction.

Table 5 Number of unrealistic estimations with average localization technique

\begin{tabular}{llllllll}
\hline & Test 1 & Test 2 & Test 3 & Test 4 & Test 5 & Test 6 & Total \\
\hline On Wall & 4 & 4 & 3 & 7 & 10 & 2 & 30 \\
Wrong Side & 1 & 5 & 8 & 2 & 1 & 2 & 19 \\
Too Close & 10 & 4 & 13 & 8 & 8 & 7 & 50 \\
Total Error & 15 & 13 & 24 & 17 & 19 & 11 & 99 \\
Total Estimations & 43 & 43 & 53 & 48 & 53 & 42 & 282 \\
Percentage Error & $35 \%$ & $30 \%$ & $45 \%$ & $35 \%$ & $36 \%$ & $26 \%$ & $35 \%$ \\
\hline
\end{tabular}




\section{BIM constraints}

The building information model can be used for supplement data to help localize a user. In Simultaneous Localization and Mapping (SLAM), the algorithm needs to both estimate the landmarks as well as estimate the location. Having to estimate the landmarks can potentially put more error in the true location, since the estimation is based on estimations. However, in a BIM, the landmarks are known, thus reducing the complexity in the algorithm and the error in true location. In addition to know landmarks, BIM provides context aware information, which is valuable in helping the localization algorithm. This context aware information is also used to put constraints on the estimation. For example, Table 5 displays the number of estimations in unrealistic places (errors) before the BIM constraints were in place when using just the tag data. Unrealistic places include on the wrong side of the wall, directly placed on the wall, and too close to the wall. An estimate was determined to be too close to the wall if the estimate places it less than $0.5 \mathrm{~m}(0.4 \mathrm{~m}$ for the width of the cart and $0.1 \mathrm{~m}$ for leeway) any wall. The experiment was repeated for the intersection approach (Table 6) and the distance equation approach (Table 7).

The results show that the total unrealistic estimations for the average, intersection and multilateration algorithms were 35,45 and $64 \%$ respectively. Therefore, implementing BIM constraints in location algorithm would play an important factor in increasing the accuracy location estimation by reducing the error to up to $64 \%$. Figs. 12, 13 and 14 shows the comparison of the paths of the different approaches with and without the BIM constraints.

\section{System accuracy}

The intersection and distance equation approaches have each been implemented to test the accuracy of the system, displayed in Tables 8 and 9. They are segregated into the error of the $\mathrm{x}, \mathrm{y}$ and radial directions. The radial direction is the hypotenuse between the corresponding $\mathrm{x}$ and $\mathrm{y}$ values from the cart. Table 8 uses the building as the coordinate reference, and Table 9 used the cart as the coordinate axis. Significantly, the total system accuracy for the

Table 6 Number of unrealistic estimations with the intersection algorithm

\begin{tabular}{llllllll}
\hline & Test 1 & Test 2 & Test 3 & Test 4 & Test 5 & Test 6 & Total \\
\hline On Wall & 0 & 0 & 0 & 0 & 0 & 0 & 0 \\
Wrong Side & 9 & 10 & 0 & 7 & 9 & 4 & 39 \\
Too Close & 13 & 10 & 17 & 17 & 16 & 16 & 89 \\
Total Error & 22 & 20 & 17 & 24 & 25 & 20 & 128 \\
Total Estimations & 43 & 43 & 53 & 48 & 53 & 42 & 282 \\
Percentage Error & $51 \%$ & $47 \%$ & $32 \%$ & $50 \%$ & $47 \%$ & $48 \%$ & $45 \%$ \\
\hline
\end{tabular}

Table 7 Number of unrealistic estimations with the multilateration algorithm

\begin{tabular}{llllllll}
\hline & Test 1 & Test 2 & Test 3 & Test 4 & Test 5 & Test 6 & Total \\
\hline On Wall & 0 & 0 & 0 & 0 & 0 & 0 & 0 \\
Wrong Side & 17 & 17 & 25 & 28 & 19 & 17 & 123 \\
Too Close & 11 & 5 & 12 & 3 & 16 & 11 & 58 \\
Total Error & 28 & 22 & 37 & 31 & 35 & 28 & 181 \\
Total Estimations & 43 & 43 & 53 & 48 & 53 & 42 & 282 \\
Percentage False & $65 \%$ & $51 \%$ & $70 \%$ & $65 \%$ & $66 \%$ & $67 \%$ & $64 \%$ \\
\hline
\end{tabular}

intersection approach is $1.66 \mathrm{~m}$ and system accuracy for the distance equation approach is $1.75 \mathrm{~m}$. Both approaches are well within the $3 \mathrm{~m}$ accuracy goal, validating that BIM can drastically improve the accuracy of indoor localization techniques.

According to Table 8, the greatest amount of error in both approaches happen when the cart is turning. This may be because of the corners of the corridor not having many tags in view or the speed of the cart turning may affect the ability to read tags. Table 9 shows that the

Table 8 Root mean square error of system, building as reference

\begin{tabular}{|c|c|c|c|c|c|c|c|}
\hline \multicolumn{4}{|c|}{ Intersection approach } & \multicolumn{4}{|c|}{ Distance equation approach } \\
\hline Total & $x$ & Y & Radial & Total & $x$ & Y & Radial \\
\hline Test 1 & 1.27 & 0.97 & 1.60 & Test 1 & 1.08 & 1.31 & 1.69 \\
\hline Test 2 & 0.98 & 1.11 & 1.48 & Test 2 & 1.17 & 0.89 & 1.47 \\
\hline Test 3 & 1.53 & 1.11 & 1.89 & Test 3 & 1.43 & 1.22 & 1.88 \\
\hline Test 4 & 1.11 & 1.12 & 1.57 & Test 4 & 1.38 & 1.21 & 1.84 \\
\hline Test 5 & 0.91 & 1.40 & 1.67 & Test 5 & 1.54 & 0.92 & 1.79 \\
\hline Test 6 & 1.17 & 1.20 & 1.69 & Test 6 & 1.63 & 1.44 & 2.17 \\
\hline Average & 1.23 & 1.48 & 1.92 & Average & 1.37 & 1.16 & 1.81 \\
\hline Turning & $x$ & Y & Radial & Turning & $x$ & Y & Radial \\
\hline Test 1 & 1.55 & 1.30 & 2.02 & Test 1 & 1.07 & 1.68 & 1.99 \\
\hline Test 2 & 1.56 & 1.12 & 1.92 & Test 2 & 1.48 & 1.10 & 1.84 \\
\hline Test 3 & 0.83 & 1.22 & 1.47 & Test 3 & 0.99 & 0.83 & 1.29 \\
\hline Test 4 & 1.68 & 1.42 & 2.20 & Test 4 & 2.15 & 1.11 & 2.42 \\
\hline Test 5 & 1.92 & 1.15 & 2.24 & Test 5 & 3.01 & 0.60 & 3.07 \\
\hline Test 6 & 1.58 & 0.80 & 1.77 & Test 6 & 2.69 & 0.69 & 2.78 \\
\hline Average & 1.52 & 1.17 & 1.94 & Average & 1.90 & 1.00 & 2.23 \\
\hline Straight & $x$ & Y & Radial & Straight & $x$ & Y & Radial \\
\hline Test 1 & 1.18 & 1.50 & 1.91 & Test 1 & 1.08 & 1.25 & 1.65 \\
\hline Test 2 & 1.22 & 0.95 & 1.55 & Test 2 & 1.12 & 0.86 & 1.41 \\
\hline Test 3 & 1.00 & 1.09 & 1.48 & Test 3 & 1.49 & 1.27 & 1.96 \\
\hline Test 4 & 1.51 & 1.08 & 1.86 & Test 4 & 1.29 & 1.22 & 1.78 \\
\hline Test 5 & 1.01 & 1.11 & 1.50 & Test 5 & 1.35 & 0.94 & 1.64 \\
\hline Test 6 & 0.78 & 1.46 & 1.66 & Test 6 & 1.43 & 1.51 & 2.08 \\
\hline Average & 1.12 & 1.20 & 1.66 & Average & 1.29 & 1.18 & 1.75 \\
\hline
\end{tabular}


Table 9 Root mean square error of system, mobile cart as reference

\begin{tabular}{|c|c|c|c|c|c|c|c|}
\hline \multicolumn{4}{|c|}{ Intersection approach } & \multicolumn{4}{|c|}{ Distance equation approach } \\
\hline & $x$ & Y & Radial & & $x$ & Y & Radial \\
\hline Test 1 & 0.72 & 1.77 & 1.91 & Test 1 & 0.78 & 1.46 & 1.65 \\
\hline Test 2 & 1.06 & 1.13 & 1.55 & Test 2 & 0.80 & 1.16 & 1.41 \\
\hline Test 3 & 1.02 & 1.08 & 1.48 & Test 3 & 1.42 & 1.35 & 1.96 \\
\hline Test 4 & 0.70 & 1.72 & 1.86 & Test 4 & 0.65 & 1.65 & 1.78 \\
\hline Test 5 & 0.68 & 1.34 & 1.50 & Test 5 & 0.62 & 1.52 & 1.64 \\
\hline Test 6 & 0.62 & 1.54 & 1.66 & Test 6 & 0.71 & 1.96 & 2.08 \\
\hline Average & 0.80 & 1.43 & 1.66 & Average & 0.83 & 1.52 & 1.75 \\
\hline
\end{tabular}

error in the $\mathrm{Y}$ direction (front and back of the cart) is significantly greater than the error of the $\mathrm{X}$ direction (left and right of the cart). This can be attributed to two reasons. The first is the fact that cart is in a corridor and thus the walls are to the left and right. This reduces the error in the $\mathrm{X}$ direction because of the constraints put in by the BIM model. The second reason is that the cart does not have an antenna facing the back, thus the RFID antennas do not have a $360^{\circ}$ view. Therefor it's biased in having more estimations in front of the cart.

\section{Discussion}

In this study, we investigated the role that Building Information Modeling (BIM) can have in common indoor localization techniques. The hypothesis is that using the information contained within a building information model can increase the accuracy of indoor positioning algorithms. As discussed before, some techniques need to estimate landmarks and the surrounding area in order to be used in the position estimations algorithms. Thus, we argue that building information models, which are becoming more prevalent in the industry, can provide that information. For example, when the algorithm estimates a landmark, that estimation will have an error associated to it, and will affect the total error when it is used to locate the position. Therefore, knowing the position of the landmarks can drastically increase the location estimation, as was shown in the results.

The approach used in this study included passive RFID technology. We demonstrated the feasibility of using RFID technology and the benefits it has, such as low maintenance and cost. Additionally, we are taking advantage of the tags that are already on various building objects from the supply chain management and installation of those objects, in which the known coordinates would be placed in the building information model. However, if building assets are not tagged with RFID, adding tags throughout the building and inputting the locations in the building information model requires potentially significant other resources, such as additional or other sensing and interface infrastructure, manual effort, and time.

Our proposed framework and method does implement passive RFID technology, however, the algorithms can be adjusted slightly to incorporate other technologies, such as WIFI, cellular, Bluetooth, or cameras, to name a few. The novel portion of the algorithms is how to integrate both the BIM data and landmark estimations to produce accurate location estimations. Significantly, the results validate the hypothesis that BIM can increase indoor localization accuracy, and show the usefulness of using BIM for indoor localization in addition to real-time visualization.

\section{Conclusions and future development}

The integration of real-time location tracking data in a BIM provides many useful applications to optimize safety, security and productivity. Integrating passive RFID technology and a BIM model into a single application and deploying it for indoor settings could play a more significant role in advancing decision making in FM. The integration provides valuable context-aware information that can be helpful for preventative maintenance. The data fusion between passive RFID technology and a BIM model serves to be an invaluable accomplishment that can be utilized for future research and applications.

The major contribution of this paper is the demonstration of how information stored within the BIM model can increase the accuracy of localization algorithms. This paper described what information can be utilized, and how that information can be used. Other contributions include the framework to use the BIM data for indoor localization, and the algorithm incorporating the data.

This research makes use of multiple localization techniques. With the framework presented, users can choose any technique and integrate the BIM mode data into it. The paper demonstrates the importance that the BIM model can pay on such techniques to provide additional context-aware information to increase the accuracy. The system accuracy was within the $3 \mathrm{~m}$ accuracy goal, validating that BIM can improve the accuracy of indoor localization techniques.

The results provided the feasibility of integrating passive RFID with the BIM for indoor settings. However, the characteristics of the passive RFID present challenges for pinpointing the true locations, such as misreads or lower RSSI than expected. Further refining is required to correct these challenges, including adjustments to the RSSI maps and the Friis forward-link equation. Adding a particle filter may help calculate the locations of the tags at a higher probability, resulting in a lower system error. More defined testing will be needed to implement additional measures. Field implementation needs to be done with large numbers of tags, and performance of the application should be 
tested in real world conditions. Eventually robot-assisted systems become available (Cicirelli et al. 2012). This research can also be extended to consider fire and rescue operations because it offers the potential for developing a tool for saving lives in the event of an emergency or providing right-time alerts (Teizer et al. 2010, Marks and Teizer 2012, Marks and Teizer 2013, Costin et al. 2015). In addition to finding tagged utilities, the location of medical personnel or equipment in hospitals, police dogs trained to find explosives in a building, and evacuation routes can all be found using indoor localization. Since RFID tags do not rely on a power supply from the facility, they keep working in the event of a power outage. This may prove crucial since the rescue team can still use the tags to guide them through the facility (heat and fire resistance of tags still needs to be researched).

\section{Competing interests}

The authors declare that they have no competing interests.

\section{Authors' contributions}

Both authors contributed extensively to the work presented in this paper. AMC reviewed and analyzed the literature, developed the model, conducted the case study, analyzed the results and drafted the manuscript. JT initiated, coordinated and supervised the entire research process of this study and edited the manuscript. Both authors read and approved the final manuscript.

\section{Acknowledgements}

This material is based upon work supported by the National Science Foundation Graduate Research Fellowship under Grant No. DGE-1148903 and Bernd Schoner from Trimble Navigation Ltd. Any opinion, findings, and conclusions or recommendations expressed in this material are those of the authors and do not necessarily reflect the views of the National Science Foundation or any other contributors.

\section{Author details}

'Georgia Institute of Technology, 790 Atlantic Dr. N.W., Atlanta, GA 30332-0355, USA. ${ }^{2}$ RAPIDS Construction Safety and Technology Laboratory, Ettlingen, Germany.

\section{Received: 21 August 2015 Accepted: 13 December 2015}

Published online: 22 December 2015

\section{References}

Ahson, S, \& llyas, M. (2008). RFID Handbook: Applications, Technology, Security and Privacy. Boca Raton: CRC Press.

Akcamete, A, Akinci, B, Garrett, J. (2010). Potential utilization of building information models for planning maintenance activities. Proc., Int. Con. on Computing in Civil and Building Engineering, Nottingham. 151-157.

Anumba, C \& Aziz, Z. (2006). Case studies of intelligent context-aware services delivery in AEC/FM, 13th EG-ICE Workshop, Ascona, Switzerland, 23-31

Becerik-Gerber, B, Jazizadeh, F, Li, N, \& Calis, G. (2012). Application Areas and Data Requirements for BIM-Enabled Facilities Management. Journal of Construction Engineering and Management, 138(3), 431-442.

Behzadan, AH, Aziz, Z, Anumba, CJ, \& Kamat, VR. (2008). Ubiquitous location tracking for context-specific information delivery on construction sites. Automation in Construction, 17, 737-748.

Bekkali, A, Sanson, H, Matsumoto, M. (2007). RFID indoor positioning based on probabilistic RFID map and Kalman filtering. 3rd IEEE Int. Con. on Wireless and Mobile Computing, Networking and Communications. New York City.

Bernoulli, T, Glanzer, G, Wießflecker, T, Walder, U. (2010). Infrastructurless Indoor Positioning System for First Responders, Proc. of the 7th International ISCRAM Conference. Seattle.

Bhaskar, S. (2007). Is RFID Technology Secure and Private? RFID handbook: Applications, technology, security and privacy (pp. 469-484). Boca Raton: Taylor \& Francis.
Bouet, M, \& dos Santos, AL. (2008). RFID tags: Positioning principles and localization techniques. IEEE 1st IFIP Wireless Days. United Arab Emirates, 1-5.

buildingSMART (2013). IFC Overview Summary. IFC Overview, <http://www. buildingsmart-tech.org/specifications/ifc-overview> (Last Accessed Nov. 20, 2013)

Bohn, JS, \& Teizer, J. (2010). Benefits and barriers of construction project monitoring using hi-resolution automated cameras. ASCE Journal of Construction Engineering and Management, Reston, Virginia, 136(6), 632-640.

Chen, A. Y, Peña-Mora, F, Plans, AP, Mehta, SJ, \& Aziz, Z. (2012). Supporting Urban Search and Rescue with digital assessments of structures and requests of response resources. Advanced Engineering Informatics, Elsevier, 26(4), 833-845.

Cheng, M, \& Chang, N (2011). Radio Frequency Identification (RFID) integrated with Building Information Model (BIM) for open-building life cycle information management. Proceedings of the 28th ISARC, Seoul. 485-490.

Cheng, T, \& Teizer, J. (2013a). Real-time Resource Location Data Collection and Visualization Technology for Construction Safety and Activity Monitoring Applications. Automation in Construction, Elsevier, 23, 3-15.

Cheng, T, \& Teizer, J (2013b). Modeling Tower Crane Operator Visibility to Minimize the Risk of Limited Situational Awareness. ASCE Journal of Computing in Civil Engineering, 28(3), 04014004. doi:10.1061/(ASCE)CP.19435487.0000282.

Cheng, T, Migliaccio, GC, Teizer, J, \& Gatti, UC (2013c). Data Fusion of Real-time Location Sensing (RTLS) and Physiological Status Monitoring (PSM) for Ergonomics Analysis of Construction Workers. ASCE Journal of Computing in Civil Engineering, 27(3), 320-335. doi:10.1061/(ASCE)CP.1943-5487.0000222.

Cheng, T, Teizer, J, Migliaccio, GC, \& Gatti, UC. (2013d). Automated Task-level Productivity Analysis through Fusion of Real Time Location Sensors and Worker's Thoracic Posture Data. Automation in Construction, Elsevier, 29, 24-39.

Cheng, T, Venugopal, M, Teizer, J, \& Vela, PA. (2011). Performance evaluation of ultra wideband technology for construction resource location tracking in harsh environments. Automation in Construction, Elsevier, 20, 1173-1184.

Cicirelli, G, Milella, A, \& Donato, DP. (2012). RFID tag localization by using adaptive neurofuzzy inference for mobile robot applications. Industrial Robot, 39(4), 340-348.

Choi, J, Lee, H, Elmasri, R, Engels, DW. (2009). Localization Systems Using Passive UHF RFID, 5th Int. Joint Con. on INC, IMS and IDC. Seoul. 1727-1732.

Costin, A, Pradhananga, N, Teizer, I (2012a). Integration of Passive RFID Location Tracking in Building Information Models (BIM), EG-ICE, Int. Workshop, Herrsching. 4-6.

Costin, A, Pradhananga, N, \& Teizer, J. (2012b). Leveraging passive RFID technology for construction resource field mobility and status monitoring in a high-rise renovation project. Automation in Construction, Elsevier, 24, 1-15.

Costin, A, Pradhananga, N, Teizer, J. (2014). Passive RFID and BIM for real-time visualization and location tracking. In Proceedings of the Construction Research Congress, 169-178.

Costin, A, Shaak, A, \& Teizer, J. (2013). Development of a Navigational Algorithm in BIM for Effective Utility Maintenance Management of Facilities Equipped with Passive RFID (pp. 653-660). Los Angeles: ASCE Computing In Civil Engineering.

Costin, A, \& Teizer, J. (2014). Utilizing BIM for Real-Time Visualization and Indoor Localization of Resources. In Computing in Civil and Building Engineering. ASCE, 649-665. http://dx.doi.org/10.1061/9780784413616.081.

Costin, AM, Teizer, J, \& Schoner, B (2015). RFID and BIM-enabled worker location tracking to support real-time building protocol and data visualization. ITcon, 20, 495-517. http://www.itcon.org/2015/29.

Deyle, T, Kemp, CC, \& Reynolds, MS. (2008). Probabilistic UHF RFID tag pose estimation with multiple antennas and a multipath RF propagation model (pp. 1379-1384). Nice: IEEE/RSJ Int. Con on Intelligent Robots and Systems.

Durrant-Whyte, H, \& Bailey, T. (2006). Simultaneous localization and mapping: part I, Robotics \& Automation Magazine. IEEE, 13(2), 99-110.

Eastman, C, Teicholz, P, Sacks, R, \& Liston, K. (2011). BIM Handbook - a guide to building information modeling. Hoboken: Wiley.

Ergen, E, Akinci, B, East, B, \& Kirby, J. (2007a). Tracking components and maintenance history within a facility utilizing radio frequency identification technology. ASCE Journal of Computing in Civil Engineering, Reston, Virginia, 21, 11-20.

Ergen, E, Akinci, B, \& Sacks, R. (2007a). Life-cycle data management of engineered-to-order components using radio frequency identification. Advanced Engineering Informatics, 21, 356-366.

Ergen, E, Guven, G, Sariel-Talay, S, \& Avdan, G. (2011). Local information access for search and rescue using wireless data storage mediums. ASCE Journal of Computing in Civil Engineering, Reston, Virginia, 25(4), 263-274. 
Fink, A, \& Beikirch, H (2011). Analysis of RSS-based location estimation techniques in fading environments, Indoor Positioning and Indoor Navigation (IPIN), Sept 21-23, 1-6. http://dx.doi.org/10.1109/IPIN.2011.6071943.

Goodrum, P, McLaren, M, \& Durfee, A. (2006). The application of active radio frequency identification technology for tool tracking on construction job sites. Automation in Construction, Elsevier, 15, 292-302.

Haehnel, D, Burgard, W, Fox, D, Fishkin, K, \& Philipose, M. (2004). Mapping and localization with RFID technology (pp. 1015-1020). Barcelona: IEEE Int. Con. on Robotics and Automation.

Jaselskis, EJ, \& El-Misalami, T. (2003). Implementing radio frequency identification in the construction process. Journal of Construction Engineering and Management, 129(6), 680-688.

Kean, T. (2011). Current Trends and Future Outlook Examining the IFMA industry trend report. Military Engineer, 103(673), 67-68.

Kelm, A, Laußat, L, Meins-Becker, A, Platz, D, Khazaee, M, Costin, A, et al. (2013). Mobile passive radio frequency identification (RFID) portal for automated and rapid control of Personal Protective Equipment (PPE) on construction sites. Automation in Construction, Elsevier, 36, 38-52.

Khoury, H, \& Kamat, V. (2009). Evaluation of position tracking technologies for user localization in indoor construction environments. Automation in Construction, 18, 444-457.

Kropp, C, Koch, C, König, M (2014). Drywall State Detection in Image Data for Automatic Indoor Progress Monitoring, International Conference on Computing in Civil and Building Engineering, 347-354. http://dx.doi.org/10.1061/9780784413616.044.

Lee, J-K, Eastman, CM, Lee, J, Kannala, M, \& Jeong, Y-S. (2010). Computing walking distances within buildings using the universal circulation network. Environment and Planning B: Planning and Design, 37(4), 628-645.

Lee, S, \& Akin, Ö. (2009). Shadowing tradespeople: Inefficiency in maintenance fieldwork. Automation in Construction, Elsevier, 18(5), 536-546.

Li, N, Becerik-Gerber, B, Krishnamachari, B, \& Soibelman, L. (2014). A BIM centered indoor localization algorithm to support building fire emergency response operations. Automation in Construction, 42, 78-89.

Li, N, Li, S, Becerik-Gerber, B, \& Calis, G. (2011). Design and evaluation of algorithm and deployment parameters for an RFID based indoor location sensing solution (pp. 77-84). Miami: International Conference on Computing in Civil Engineering

Liu, T, Carlberg, M, Chen, G, Chen, J, Kua, J, \& Zakhor, A. (2010). Indoor localization and visualization using a human-operated backpack system (pp. 1-10). Zurich: International Conference on Indoor Positioning and Indoor Navigation.

Marks, E, \& Teizer, J. (2012). Safety first: real-time proactive equipment operator and ground worker warning and alert system in steel manufacturing. Iron \& Steel Technology, AIST, 9(10), 56-69.

Marks, E, \& Teizer, J. (2013). Method for Testing Proximity Detection and Alert Technology for Safe Construction Equipment Operation, Construction Management and Economics, Taylor \& Francis. Special Issue on Occupational Health and Safety in the Construction Industry, 31(6), 636-646.

McAndrew, ST, Anumba, CJ, Hassan, TM, \& Duke, AK. (2005). Potential use of real-time data capture and job-tracking technology in the field. Facilities, 23(1/2), 31-46.

McGraw-Hill. (2012). SmartMarket Report: The Business Value of BIM in North America Multi-Year Trend Analysis and User Ratings (2007-2012), Construction. New York: McGraw-Hill Construction.

Meadati, P, Irizarry, J, Akhnoukh, AK. (2010). BIM and RFID Integration: A Pilot Study, 2nd International Conference on Construction in Developing Countries, Cairo, Egypt, 570-578.

Memarzadeh, M, Golparvar-Fard, M, Niebles, J. (2012). Automated 2D detection of construction equipment and workers from site video streams using histograms of oriented gradients and colors, Automation in Construction, Elsevier, 32, 24-37.

Ogaja, CA. (2011). Applied GPS for engineers and project managers, ASCE Press.

Olatunji, OA, \& Sher, W. (2009). Process problems in facilities management: an analysis of feasibility and management indices, BuHu 9th International Postgraduate Research Conference, Greater Manchester, 199-211

Razavi, SN, Montaser, A, \& Moselhi, O. (2012). RFID deployment protocols for indoor construction. Construction Innovation, 12(2), 239-258.

Ross, W, Burns, S, \& Wu, J. (2009). Radio frequency identification tracking technology applied to testing of transportation construction materials. Journal of Transportation Research Board, 2098, 3-12.

Park, Y, Lee, JW, Kim, S (2009). Improving position estimation on RFID tag floor localization using RFID reader transmission power control, International Conference on Robotics and Biometrics, Bangkok, Thailand, 1716-1721

Pradhan, A, Ergen, E, \& Akinci, B. (2009). Technological assessment of radio frequency identification technology for indoor localization. ASCE Journal of Computing in Civil Engineering, Reston, Virginia, 23(4), 230-238.
Pradhananga, N, \& Teizer, J. (2013). Automatic spatio-temporal analysis of construction equipment operations using GPS data. Automation in Construction, Elsevier, 29, 107-122.

Pradhananga, N, \& Teizer, J (2015). Cell-based construction site simulation model for earthmoving operations using real-time equipment location data, visualization in engineering, Springer Verlag, http://dx.doi.org/10.1186/s40327-015-0025-3.

Rüppel, U, \& Stübbe, K. (2008). BIM based indoor-emergency-navigation-system for complex buildings. Tsinghua Science and Technology Journal, Tsinghua University, 13(S1), 362-367.

Sawyer, T (2014). 1-Billion Jigsaw Puzzle Has Builder Modeling Supply Chains. http://enr.construction.com/features/technologyEconst/archives/080423-1. asp, (Accessed March 28, 2014).

Sullivan, G, Pugh, R, Melendez, AP, \& Hunt, WD. (2010). Operations \& Maintenance Best Practices - A Guide to Achieving Operational Efficiency (Release 3). Prepared by PNNL for the U.S. DOE.

Taneja, S, Akcamete, A, Akinci, A, Garrett, J, Soibelman, L, \& East, E. (2012). Analysis of Three indoor localization technologies for supporting operations and maintenance field tasks. ASCE Journal of Computing in Civil Engineering, Reston, Virginia, 26(6), 708-719.

Teicholz, E. (2004). Bridging the AEC technology gap. IFMA Facility Management Journal.

Teizer, J, Cheng, T, \& Fang, Y. (2013). Location Tracking and Data Visualization Technology to Advance Construction Ironworkers' Education and Training in Safety and Productivity. Automation in Construction, Elsevier, 35, 53-68.

Teizer, J, Lao, D, Sofer, M. (2007). Rapid Automated Monitoring of Construction Site Activities using Ultra-Wideband. Proceedings of the 24th International Symposium on Automation and Robotics in Construction, Cochin, Kerala, India; September 19-21, 23-28

Teizer, J, \& Vela, PA. (2009). Personnel tracking on construction sites using video cameras. Advanced Engineering Informatics, Special Issue, Elsevier, 23(4), 452-462.

Teizer, J, Venugopal, M, \& Walia, A. (2008). Ultra wideband for automated realtime three-dimensional location sensing for workforce, equipment and material positioning and tracking, transportation research record. Journal of the Transportation Research Board, Washington D.C., 2081, 56-64.

Teizer, J, Allread, BS, Fullerton, CE, \& Hinze, J. (2010). Autonomous Pro-Active Realtime Construction Worker and Equipment Operator Proximity Safety Alert System. Automation in Construction, Elsevier, 19(5), 630-640.

Thomas, SJ, Wheeler, E, Teizer, J, \& Reynolds, MS. (2012). Quadrature Amplitude Modulated Backscatter in Passive and Semipassive UHF RFID Systems. IEEE Transactions on Microwave Theory and Techniques, 60(4), 1175-1183.

Trimble (2014). ThingMagic Mercury 6, High-Performance 4-Port Reader, Manual.

Vasenev, A, Pradhananga, N, Bijleveld, F, lonita, D, Hartmann, T, Teizer, J, Dorée, A. (2014). Information Fusion Approach to Increase the Quality of GNSS Data Sets in Construction Equipment Operations, Advanced Engineering Informatics, Elsevier, 28(4), 297-310.

Vogt, B, \& Teizer, J. (2007). Sparse Laser Scanning to Improve Off-Shore PipeFitting Productivity, Proceedings of the Construction Research Congress, Freeport, Bahamas.

Yang, J, Arif, O, Vela, PA, Teizer, J, \& Shi, Z. (2010). Tracking multiple workers on construction sites using video cameras. Special Issue of the Journal of Advanced Engineering Informatics, Elsevier, 24(4), 428-434.

Yang, J, Cheng, T, Teizer, J, Vela, PA, \& Shi, ZK. (2011). A performance evaluation of vision and radio frequency tracking methods for interacting workforce. Advanced Engineering Informatics, Elsevier, 25(4), 736-747.

Yang, J, Vela, PA, Teizer, J, \& Shi, ZK. (2014). Vision-based crane tracking for understanding construction activity. ASCE Journal of Computing in Civil Engineering, Reston, Virginia, 28(1), 103-112.

Wang, H. (2011). Bayesian radio map learning for robust indoor positioning, Indoor Positioning and Indoor Navigation (IPIN), Sept. 21-23, 1-6.

Wang, L. (2008). Enhancing Construction Quality Inspection and Management Using RFID Technology. Automation in Construction, Elsevier, 17, 467-479.

Wang, X, Bischoff, O, Laur, R, \& Paul, S. (2009). Localization in wireless ad-hoc sensor networks using multilateration with RSSI for logistic applications. Procedia Chemistry, 1(1), 461-464.

Wing, R. (2006). RFID application in construction and facilities management. ITcon, 11, 711-721.

Xie, H, Shi, W, Issa, R. (2010). Implementation of BIM/RFID in Computer-Aided Design-Manufacturing-Installation Process, 3rd IEEE International on Conference Computer Science and Information Technology. Chengdu. 107-111. 